Revista peruana de biología 26(4): 411 - 428 (2019) doi: http://dx.doi.org/10.15381/rpb.v26i4.17213 ISSN-L 1561-0837; eISSN: 1727-9933

Universidad Nacional Mayor de San Marcos

\section{Clave taxonómica de ninfas de Ephemeroptera (Insecta) del sudeste de Perú}

\section{TRABAJOS ORIGINALES}

$\begin{array}{ll}\text { Presentado: } & 29 / 07 / 2019 \\ \text { Aceptado: } & 04 / 09 / 2019 \\ \text { Publicado online: } & 16 / 12 / 2019\end{array}$

\section{Correspondencia:}

* Autor para correspondencia

Pablo Miñano: jminanolecaros@gmail.com

Michela Olaya: molayach@gmail.com

Ana Huamantinco: ahuamantincoa1@unmsm.edu.pe

- Laboratorio de Invertebrados Acuáticos, Oficina 228, Pabellón de investigación FCB. Ciudad Universitaria de San Marcos. Av. Venezuela Cdra. 34 s/n. Lima

- Dirección postal Pablo Miñano: Jr. Andres Torres Paz № 247. Distrito San Martin de Porres

Otros datos de los autores / biografía: ORCID Pablo Miñano: 0000-0003-2099-3006 ORCID Michela Olaya: 0000-0001-9241-3395 ORCID Ana Huamantinco: 0000-0001-6558-1326

Citación:

Miñano P., M. Olaya, A.A. Huamantinco. 2019. Clave taxonómica de ninfas de Ephemeroptera (Insecta) del sudeste de Perú. Revista peruana de biología 26(4): 411 - 428 (Diciembre 2019). doi: http:// dx.doi.org/10.15381/rpb.v26i4.17213

Palabras clave: macroinvertebrados; bentónicos; Sudamérica; efemerópteros; cuenca del Amazonas; nuevos registros, Andes; ríos tropicales; bioindicadores; biodiversidad.

Keywords: benthos; macroinvertebrates; South America; mayflies; Amazon basin; new records, Andes; tropical rivers, bioindicators; biodiversity.

\section{Taxonomic key of nymphs of Ephemeroptera (Insecta) from southeastern Peru}

\author{
Pablo Miñano*, Michela Olaya, Ana A. Huamantinco \\ Universidad Nacional Mayor de San Marcos, Facultad de Ciencias Biológicas, Laboratorio \\ de Invertebrados Acuáticos, Lima, Perú.
}

\begin{abstract}
Resumen
Ephemeroptera es un orden de insectos cuyas etapas inmaduras cumplen un importante papel ecológico en los ecosistemas acuáticos. Sin embargo, el conocimiento de su diversidad en áreas tropicales aún es escaso. Específicamente, en el Perú se han reportado 64 especies pertenecientes a 35 géneros y ocho familias. En este estudio se presenta una clave taxonómica para la determinación de géneros de ninfas de Ephemeroptera de la vertiente suroriental de los Andes del Perú. Las ninfas revisadas fueron recolectadas en las quebradas de los departamentos de Cusco y Madre de Dios, dentro de un gradiente altitudinal de $180-4411 \mathrm{~m}$, en los años 2012, 2013, 2015 y 2017. Se utilizaron tres metodologías de muestreo: recolección manual, red Surber y colonización de paquetes de hojas. Se reconocieron 48 géneros de ocho familias en 36 quebradas dentro de este gradiente. Seis géneros son nuevos registros para el Perú: Prebaetodes, Zelusia, Meridialaris, Tricorythopsis, Homoeoneuria y Hubbardipes.
\end{abstract}

\section{Abstract}

Ephemeroptera is an insect order of whose immature stages play an important ecological role in aquatic ecosystems. However, its diversity knowledge in tropical areas is scarce. In particular, only 64 species of 35 genera and eight families have been reported in Peru. In this work, we provide a general key of Ephemeroptera nymphs for southeastern Peru. Nymphs were collected from streams of Cusco and Madre de Dios departments, within an altitudinal gradient of $180-4411 \mathrm{~m}$, in 2012, 2013, 2015 and 2017 years. The samples were obtained by manual collection, using a Surber net and by colonization of leaf packs. We recognized 48 genera within eight families, coming from 36 streams within the gradient. Six genera are new records to Peru: Prebaetodes, Zelusia, Meridialaris, Tricorythopsis, Homoeoneuria and Hubbardipes. 


\section{Introducción}

La vertiente suroriental de los Andes del Perú presenta un gradiente altitudinal que abarca desde los 180 $\mathrm{m}$ hasta los $6372 \mathrm{~m}$ de altitud, incluyendo la llanura amazónica, ceja de selva y alta montaña (ONERN 1976). La importancia ecológica y económica de un gradiente altitudinal está siendo cada vez más reconocida por la gran diversidad de especies y hábitats que puede albergar (Buytaert et al. 2006, Jacobsen 2008) y, en especial, por la diversidad de los ecosistemas acuáticos de montaña (Anderson et al. 2011). Entre la biodiversidad presente en estos ecosistemas, los macroinvertebrados bentónicos son un grupo de organismos de gran importancia ecológica (Jacobsen 2008) y desempeñan un papel esencial en el reciclaje de nutrientes como enlaces importantes en las cadenas alimenticias (Santos 2016).

Uno de los grupos de macroinvertebrados bentónicos menos estudiados son los insectos acuáticos del orden Ephemeroptera. Las ninfas de los efemerópteros cumplen un rol fundamental dentro de las comunidades de los ecosistemas lóticos, debido a su diversidad, abundancia y función en el ciclo de nutrientes (Grant 2001, Domínguez et al. 2006, Menetrey et al. 2007, Brittain \& Sartori 2009). Además, su sensibilidad a la contaminación los convierte en apropiados bioindicadores de la calidad del agua (Flowers \& De la Rosa 2010). Actualmente, el orden está representado por 42 familias con 450 géneros y más de 3500 especies válidas en el mundo (Sartori \& Brittain 2015). Sin embargo, aún faltan muchas especies por descubrir, principalmente en las zonas tropicales, donde ocurre la más alta diversidad genérica (BarberJames et al. 2008).
En años recientes, el conocimiento del orden en América Latina ha incrementado gracias a estudios entomológicos y al aumento de inventarios de biodiversidad, que han brindado material de alta calidad para la descripción de nuevos géneros y especies (Lima et al. 2016, Cruz et al. 2017, Salles et al. 2018, Cruz et al. 2018, Cruz \& Hamada 2019). En América del Sur existen hasta la fecha, registros de 14 familias, aproximadamente 112 géneros y 671 especies (Domínguez et al. 2019). Mientras que, en el Perú se han registrado solo 64 especies en 35 géneros y ocho familias. El número de registros de la fauna de efemerópteros comparado con los demás países sudamericanos refleja problemas asociados con estudios taxonómicos, registros no publicados y falta de preocupación por su conservación (Pescador et al. 2001, Domínguez et al. 2019). Por tanto, proporcionar metodologías, guías, claves taxonómicas, entre otras herramientas se torna fundamental para mejorar el entendimiento de estos insectos acuáticos. En tal sentido, este estudio presenta una clave taxonómica de las ninfas de Ephemeroptera en la vertiente suroriental de los Andes del Perú, con base en los trabajos de Domínguez et al. (2006), Domínguez y Fernández (2009) y Salles et al. (2018). Cabe mencionar que, en este trabajo se considera vertiente suroriental de los Andes a la zona de la cordillera de los Andes situada entre el Sur de Perú y el Oeste de Brasil y Bolivia.

\section{Material y métodos}

El material revisado consta de ninfas de efemerópteros recolectadas durante los años 2012, 2013, 2015 y 2017, en quebradas de los departamentos de Cusco y Madre de Dios (Fig. 1), dentro de un gradiente altitu-

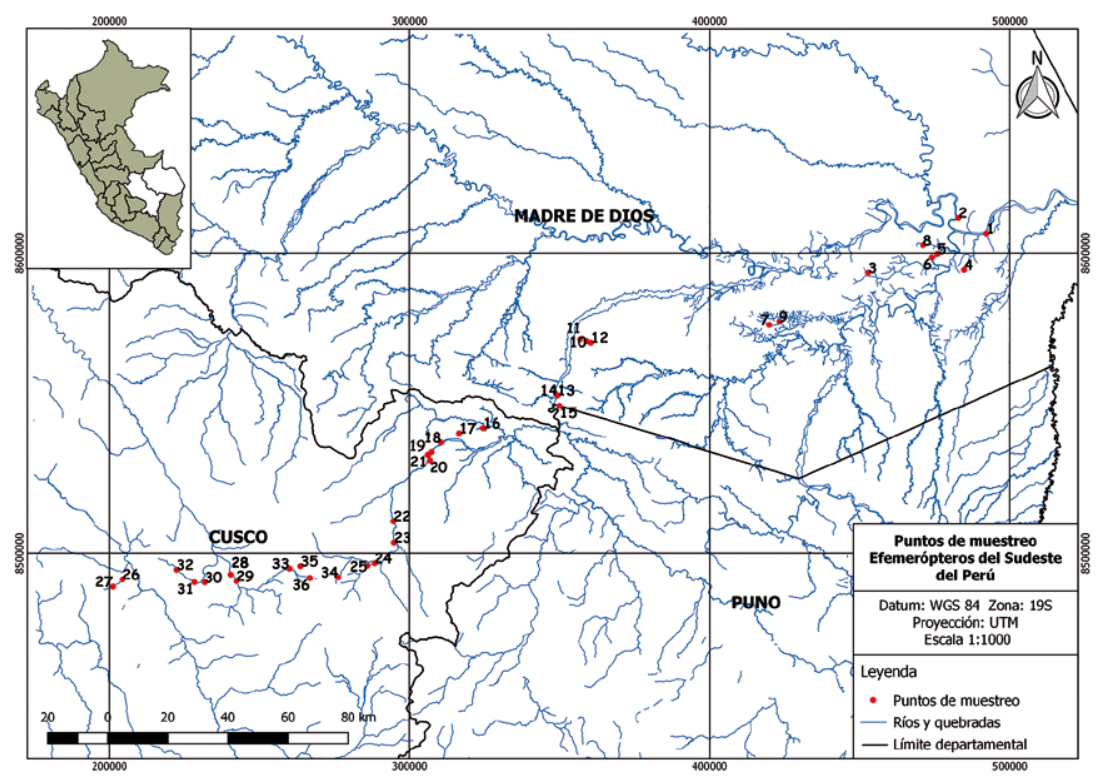

Figura 1. Ubicación geográfica de las quebradas muestreadas en el Sudeste del Perú. 1. Fundo Concepción, 2. Cachuela, 3. Maquisapayoc, 4. Otorongo, 5. Infierno 3, 6. Infierno 5, 7. Santo Rosario, 8. La Colina, 9. San Juan, 10. Central Santa Rosa, 11. West Santa Rosa, 12. East Santa Rosa, 13. Puerto Mazuco, 14. Puerto Mazuco Trib., 15. Palmera, 16. Limonchayoc, 17. Limonchayoc arriba, 18. Quincemil, 19. Oroyita, 20. Yanamayo Grande, 21. Chilimayo, 22. Chaupichaca, 23. Ttio, 24. Nihuasamana, 25. Sahuancay, 26. Lucre, 27. Lucre arriba, 28. Chacachimpa, 29. Mapocho, 30. Yuncayoc, 31. Catcamayo 2, 32. Catcamayo 1, 33. Jollepunco, 34. Palquella, 35. Ajopampa, 36. Mapocho arriba. 
dinal de 180 m hasta $4411 \mathrm{~m}$ (Tabla 1). Las quebradas atraviesan zonas de vida dentro de la Región Latitudinal Subtropical: bosque húmedo (bh-S), bosque muy húmedo (bmh-S), bosque pluvial (bp-S), bosque seco Montano Bajo (bs-MBS) y páramo pluvial Subalpino (pp-SaS) (ONERN 1976).

El material estudiado procede de los muestreos desarrollados durante 2012-2013 en la investigación "Impacto de la Carretera Interoceánica sobre la calidad del agua en la región de las nacientes Andino-Amazónicas del este, Cusco-Madre de Dios, Perú" proyecto concebido y ejecutado por Amazon Center for Environmental Edu- cation and Research (ACEER) en asociación con Stroud Water Research Center. En este estudio se aplicaron dos métodos de recolección: (1) recolecta manual de ninfas en todos los tipos de hábitats, realizada por entomólogos acuáticos de Stroud Water Research Center, y (2) la ubicación de paquetes artificiales de hojas (Inga Mill) por cuatro semanas, para la colonización de macroinvertebrados (STROUD 2010). También se utilizó material de las tesis de Barra (2015) y Pinto (2018), estudios que usaron la metodología de paquetes de hojas en quebradas de Madre de Dios. Asimismo, se incluyó el material de la tesis de Miñano (2017), recolectado en quebradas

Tabla 1. Ubicación de las quebradas muestreadas en el Sudeste del Perú.

\begin{tabular}{|c|c|c|c|c|c|}
\hline \multirow{2}{*}{ Quebrada } & \multirow{2}{*}{ Código } & \multirow{2}{*}{ Departamento } & \multirow{2}{*}{ Altitud (m) } & \multicolumn{2}{|c|}{ Coordenadas Geográficas } \\
\hline & & & & Latitud & Longitud \\
\hline Fundo Concepción & ATI & Madre de Dios & 180 & $12^{\circ} 35^{\prime} 58.2000^{\prime \prime} \mathrm{S}$ & $69^{\circ} 04^{\prime} 19.7598^{\prime \prime} \mathrm{W}$ \\
\hline Cachuela & CAC & Madre de Dios & 200 & $12^{\circ} 33^{\prime} 09.9252^{\prime \prime S}$ & $69^{\circ} 09^{\prime} 27.5766^{\prime \prime W}$ \\
\hline Maquisapayoc & MAQ & Madre de Dios & 217 & $12^{\circ} 43^{\prime} 22.4400^{\prime \prime S}$ & $69^{\circ} 26^{\prime} 04.2720^{\prime \prime} \mathrm{W}$ \\
\hline Otorongo & ОТО & Madre de Dios & 222 & $12^{\circ} 42^{\prime} 34.1964^{\prime \prime S}$ & $69^{\circ} 08^{\prime} 26.2459^{\prime \prime} \mathrm{W}$ \\
\hline Infierno 3 & IN3 & Madre de Dios & 227 & $12^{\circ} 39^{\prime} 57.6000^{\prime \prime S}$ & $69^{\circ} 13^{\prime} 22.0080^{\prime \prime} \mathrm{W}$ \\
\hline Infierno 5 & IN5 & Madre de Dios & 227 & $12^{\circ} 40^{\prime} 35.4000^{\prime \prime S}$ & $69^{\circ} 14^{\prime} 21.1200^{\prime \prime W}$ \\
\hline Santo Rosario & SRO & Madre de Dios & 227 & $12^{\circ} 52^{\prime} 43.7520^{\prime \prime S}$ & $69^{\circ} 44^{\prime} 22.5240^{\prime \prime} \mathrm{W}$ \\
\hline La Colina & LAC & Madre de Dios & 230 & $12^{\circ} 38^{\prime} 24.9000^{\prime \prime S}$ & $69^{\circ} 16^{\prime} 00.2640^{\prime \prime} \mathrm{W}$ \\
\hline San Juan & CON & Madre de Dios & 248 & $12^{\circ} 51^{\prime} 51.2172 " \mathrm{~S}$ & $69^{\circ} 42^{\prime} 29.9022^{\prime \prime W}$ \\
\hline Central Santa Rosa & CSR & Madre de Dios & 308 & $12^{\circ} 55^{\prime} 31.3320^{\prime \prime S}$ & $70^{\circ} 18^{\prime} 00.5400^{\prime \prime W}$ \\
\hline West Santa Rosa & WSR & Madre de Dios & 308 & $12^{\circ} 55^{\prime} 13.2960^{\prime \prime S}$ & $70^{\circ} 19^{\prime} 12.5400^{\prime \prime} \mathrm{W}$ \\
\hline East Santa Rosa & ESR & Madre de Dios & 309 & $12^{\circ} 55^{\prime} 48.5760^{\prime \prime} \mathrm{S}$ & $70^{\circ} 17^{\prime} 13.5960^{\prime \prime} \mathrm{W}$ \\
\hline Puerto Mazuco & PMZ & Madre de Dios & 321 & $13^{\circ} 05^{\prime} 19.3200^{\prime \prime} \mathrm{S}$ & $70^{\circ} 23^{\prime} 24.7560^{\prime \prime} \mathrm{W}$ \\
\hline Puerto Mazuco Trib. & PMT & Madre de Dios & 326 & $13^{\circ} 05^{\prime} 19.5360^{\prime \prime} \mathrm{S}$ & $70^{\circ} 23^{\prime} 26.0520^{\prime \prime} \mathrm{W}$ \\
\hline Palmera & PAL & Madre de Dios & 361 & $13^{\circ} 07^{\prime} 12.8640^{\prime \prime S}$ & $70^{\circ} 23^{\prime} 03.0120^{\prime \prime} \mathrm{W}$ \\
\hline Limonchayoc & LIM & Cusco & 476 & $13^{\circ} 11^{\prime} 08.6000^{\prime S}$ & $70^{\circ} 37^{\prime} 10.6000^{\prime \prime} \mathrm{W}$ \\
\hline Limonchayoc arriba & LMA & Cusco & 593 & $13^{\circ} 12^{\prime} 03.2000^{\prime S}$ & $70^{\circ} 41^{\prime} 37.7000^{\prime \prime} \mathrm{W}$ \\
\hline Quincemil & QUI & Cusco & 678 & $13^{\circ} 13^{\prime} 40.7640 " \mathrm{~S}$ & $70^{\circ} 44^{\prime} 54.3840^{\prime \prime} \mathrm{W}$ \\
\hline Oroyita & ORO & Cusco & 695 & $13^{\circ} 15^{\prime} 23.3640^{\prime \prime S}$ & $70^{\circ} 46^{\prime} 45.2640^{\prime \prime} \mathrm{W}$ \\
\hline Yanamayo Grande & YMG & Cusco & 731 & $13^{\circ} 16^{\prime} 54.8000^{\prime \prime} \mathrm{S}$ & $70^{\circ} 47^{\prime} 01.8000^{\prime \prime} \mathrm{W}$ \\
\hline Chilimayo & $\mathrm{CHI}$ & Cusco & 803 & $13^{\circ} 15^{\prime} 54.0000 " \mathrm{~S}$ & $70^{\circ} 47^{\prime} 25.9000^{\prime \prime} \mathrm{W}$ \\
\hline Chaupichaca & $\mathrm{CHA}$ & Cusco & 1453 & $13^{\circ} 27^{\prime} 50.8000^{\prime \prime} \mathrm{S}$ & $70^{\circ} 53^{\prime} 50.8000^{\prime \prime} \mathrm{W}$ \\
\hline Ttio & TIO & Cusco & 1912 & $13^{\circ} 31^{\prime} 42.1680^{\prime \prime S}$ & $70^{\circ} 53^{\prime} 46.5000^{\prime \prime} \mathrm{W}$ \\
\hline Nihuasamana & $\mathrm{NIH}$ & Cusco & 2638 & $13^{\circ} 35^{\prime} 24.0000^{\prime \prime S}$ & $70^{\circ} 57^{\prime} 21.7800^{\prime \prime} \mathrm{W}$ \\
\hline Sahuancay & $\mathrm{SAH}$ & Cusco & 2917 & $13^{\circ} 35^{\prime} 50.4600^{\prime \prime S}$ & $70^{\circ} 58^{\prime} 44.2920^{\prime \prime} \mathrm{W}$ \\
\hline Lucre & LUC & Cusco & 3108 & $13^{\circ} 37^{\prime} 53.5000^{\prime \prime} \mathrm{S}$ & $71^{\circ} 43^{\prime} 57.8000^{\prime \prime} \mathrm{W}$ \\
\hline Lucre arriba & LUA & Cusco & 3259 & $13^{\circ} 39^{\prime} 10.3000^{\prime \prime S}$ & $71^{\circ} 45^{\prime} 44.6000^{\prime \prime} \mathrm{W}$ \\
\hline Chacachimpa & $\mathrm{CHC}$ & Cusco & 3522 & $13^{\circ} 37^{\prime} 13.9000^{\prime \prime S}$ & $71^{\circ} 23^{\prime} 58.6000^{\prime \prime} \mathrm{W}$ \\
\hline Mapocho & MAP & Cusco & 3575 & $13^{\circ} 38^{\prime} 24.6000^{\prime \prime S}$ & $71^{\circ} 22^{\prime} 54.8000^{\prime \prime} \mathrm{W}$ \\
\hline Yuncayoc & YUN & Cusco & 3653 & $13^{\circ} 38^{\prime} 34.4760^{\prime \prime} \mathrm{S}$ & $71^{\circ} 28^{\prime} 47.6760^{\prime \prime} \mathrm{W}$ \\
\hline Catcamayo 2 & CAT2 & Cusco & 3703 & $13^{\circ} 38^{\prime} 31.3440 " \mathrm{~S}$ & $71^{\circ} 30^{\prime} 40.5000^{\prime \prime} \mathrm{W}$ \\
\hline Catcamayo 1 & CAT1 & Cusco & 3714 & $13^{\circ} 36^{\prime} 15.0480^{\prime \prime S}$ & $71^{\circ} 33^{\prime} 58.1400^{\prime \prime W}$ \\
\hline Jollepunco & $\mathrm{JOL}$ & Cusco & 4106 & $13^{\circ} 36^{\prime} 14.9000^{\prime \prime S}$ & $71^{\circ} 13^{\prime} 06.9000^{\prime \prime} \mathrm{W}$ \\
\hline Palquella & PLQ & Cusco & 4244 & $13^{\circ} 37^{\prime} 54.1200^{\prime \prime S}$ & $71^{\circ} 04^{\prime} 06.5280^{\prime \prime} \mathrm{W}$ \\
\hline Ajopampa & AJO & Cusco & 4291 & $13^{\circ} 35^{\prime} 49.7000^{\prime \prime S}$ & $71^{\circ} 11^{\prime} 02.2000^{\prime \prime} \mathrm{W}$ \\
\hline Mapocho arriba & MAA & Cusco & 4411 & $13^{\circ} 37^{\prime} 58.4000^{\prime \prime S}$ & $71^{\circ} 09^{\prime} 21.5000^{\prime \prime} \mathrm{W}$ \\
\hline
\end{tabular}


del Cusco mediante red Surber. En todos los casos, los especímenes se conservaron en etanol al 96\%. La totalidad de ninfas revisadas se encuentra depositada en el Laboratorio de Invertebrados Acuáticos, Facultad de Ciencias Biológicas, Universidad Nacional Mayor de San Marcos en Lima, Perú; en donde también se pueden consultar los detalles de los proyectos y métodos de recolección.

Las ninfas maduras y completas se determinaron taxonómicamente a nivel de género, utilizando claves taxonómicas (Domínguez et al. 2006, Domínguez \& Fernández 2009, Salles et al. 2018) y los artículos de Domínguez (1988), Molineri (2001, 2003, 2004), Molineri y Zúñiga (2004), Salles y Molineri (2006), Dias et al. (2007), Boldrini et al. (2009) y Flowers y De la Rosa (2010), Lima et al. (2019). Además, se revisó el catálogo en línea de las especies sudamericanas (Domínguez et al. 2019), "Ephemeroptera Galactica" (www.ephemeroptera-galactica. com) y la literatura especializada recopilada en la página web "Ephemeroptera of the World" (www.insecta.bio. spbu.ru/z/Eph-spp/).

Para el análisis de los especímenes, se realizó la disección y el montaje en láminas portaobjetos de las piezas bucales, patas y branquias; posteriormente, se tomaron fotografías usando un microscopio compuesto Alpha Optics BM-2000. También, se fotografiaron los individuos completos utilizando un microscopio estereoscópico Greenough Leica S9 i con una cámara digital integrada CMOS de $10 \mathrm{Mp}$, a través del programa Leica Application Suite V4.12.0 (Versión 2017). A partir de las fotografías de los caracteres morfológicos se realizaron dibujos a mano. También, se elaboraron esquemas digitalmente según la técnica de Coleman $(2003,2006)$. Luego, ambas ilustraciones se editaron en el software Photoshop CS (18.1.0) para producir imágenes de óptima calidad.

Para la preparación de la clave se usó una combinación de caracteres diagnósticos y típicos de las familias y géneros. La terminología usada para estos caracteres es la propuesta por Domínguez et al. (2006) y Salles et al. (2018). Es preciso mencionar que en este trabajo se considera proyección posterolateral de un segmento abdominal a la notoria prolongación de su tegumento, similar a una espina. Asimismo, se utiliza el término "espina" en referencia a una evaginación del tegumento, mientras que "seta", a una proyección esclerotizada del tegumento a manera de pelo.

\section{Resultados}

Dentro del gradiente estudiado se hallaron 43 géneros de ocho familias. Para la familia Baetidae se determinaron 14 géneros (Fig. 127-140); para Leptophlebiidae 13 géneros (Fig. 114-126); para Leptohyphidae, siete géneros (Fig. 107-113); para Polymitarcyidae, tres géneros (Fig. 103-105); para Euthyplociidae y Oligoneuriidae, dos géneros por cada una (Fig. 99-102); y para Coryphoridae y Caenidae, un género por cada una (Fig. 98 y Fig. 106).

Más del 75\% de los géneros determinados se encontraron en altitudes relativamente bajas (Tabla 2). Considerando los estudios de Domínguez et al. (2019) y Sweeney et al. (2009) en Madre de Dios, registramos seis géneros nuevos para el Perú: Prebaetodes, Zelusia, Meridialaris, Tricorythopsis, Homoeoneuria y Hubbardipes. Por otro lado, se precisa que los géneros Allenhyphes, Brasilocaenis, Paracloeodes, Paramaka y Traverella no se hallaron en el material revisado; sin embargo, son incluidos en la clave por haber sido reportados dentro de la zona de estudio (Sweeney et al. 2009). De manera que, la presente clave permite la determinación taxonómica de 48 géneros en total.

Clave taxonómica de ninfas del orden Ephemeroptera del sudeste de Perú

(Adaptada de Domínguez et al. 2006, Domínguez y Fernández 2009)

1a. Vértex de la cabeza con un par de tubérculos delgados ubicados submedialmente (Fig. 2); además tubérculos en el pronoto y tergos abdominales I-III y VII-IX (Fig. 3).

\section{CORYPHORIDAE}

Coryphorus Peters, 1981 (Fig. 98)

1b.Vértex de la cabeza sin tubérculos, pueden tener o no tubérculos sobre el abdomen.

2

2a. Base de las maxilas con un penacho de branquias (Fig. 5); fémures anteriores con una doble hilera de largas setas sobre el lado interno (Fig. 4); con 2 o 3 filamentos caudales. OLIGONEURIIDAE

2b. Base de las maxilas sin un penacho de branquias; fémures anteriores sin doble hilera de largas setas sobre el lado interno (si presenta, con colmillos mandibulares; Polymitarcyidae); con 3 filamentos caudales.

4

3a. Presencia de solamente dos filamentos caudales (cercos) (Fig. 7); branquia laminar pequeña y redondeada, menor a la mitad de la longitud de cada segmento (Fig. 6); tarso anterior normalmente desarrollado.

Lachlania Hagen, 1868 (Fig. 99)

3b. Presencia de tres filamentos caudales (cercos y filamento intermedio); branquia laminar alargada, tres veces más larga que su ancho, presente en los segmentos II-VII (Fig. 8); tarso anterior vestigial (Fig. 9).

Homoeoneuria Eaton, 1881 (Fig. 100)

4a. Colmillos mandibulares presentes dirigiéndose por delante de la cabeza (Fig. 10, Fig. 11, Fig. 13, Fig. 14). 5

4b. Colmillos mandibulares ausentes

9

5a. Colmillos mandibulares muy largos, en forma de hoz y con numerosas setas largas (Fig. 10). EUTHYPLOCIIDAE

5b.Colmillos mandibulares casi rectos (Fig. 11, Fig. 13, Fig. 14).

POLYMITARCYIDAE

6a. Espina de la tibia anterior aproximadamente $1 / 4$ de la longitud del tarso (Fig. 15).

Campylocia Needham \& Murphy, 1924 (Fig. 101)

6b. Espina de la tibia anterior mayor de 1/2 de la longitud del tarso (Fig. 16). $\quad$ Euthyplocia Eaton, 1871 (Fig. 102)

7a.Cabeza dorsalmente con setas cortas (Fig. 12); ápice del colmillo mandibular izquierdo con un proceso puntiagudo (Fig. 11).

Campsurus Eaton, 1868 (Fig. 103)

7b. Cabeza dorsalmente glabra, sin setas cortas; ápice del colmillo mandibular izquierdo con tres procesos puntiagudos (Fig. 14).

8a. Colmillos mandibulares, cortos y robustos, con un gran tubérculo subbasal sobre el margen medio (Fig. 13).

Asthenopus Eaton, 1871 (Fig. 104) 
Tabla 2. Distribución altitudinal de los géneros del orden Ephemeroptera del Sudeste del Perú.

\begin{tabular}{|c|c|c|}
\hline Género & Quebradas & Rango altitudinal (m) \\
\hline Americabaetis & IN5, SRO, CSR, ESR, PAL, LIM, LIA, ORO, CHI, CHA & $227-1453$ \\
\hline Andesiops & $\begin{array}{l}\text { CHA, TIO, NIH, SAH, LUC, LUA, CHC, MAP, YUN, CAT2, CAT1, JOL, PLQ, } \\
\text { AJO, MAA }\end{array}$ & $1453-4411$ \\
\hline Aturbina & IN5, ESR & $227-309$ \\
\hline Baetodes & CSR, WSR, ESR, PAL, LIM, LIA, ORO, YMG, CHI, CHA, TIO, NIH, LUA, CHC & $308-3522$ \\
\hline Callibaetis & CSR & 308 \\
\hline Camelobaetidius & ESR, PAL, LIM, LIA, QUI, YMG, CHA, TIO & $309-1912$ \\
\hline Cloeodes & SRO, LIM, LIA, ORO & $227-695$ \\
\hline Cryptonympha & SRO, ESR, LIM & $227-476$ \\
\hline Guajirolus & ATI & 180 \\
\hline Mayobaetis & YMG, $\mathrm{CHI}, \mathrm{CHA}$ & $731-1453$ \\
\hline Nanomis & ESR, YMG, CHA & $309-1453$ \\
\hline Prebaetodes & YMG, $\mathrm{CHI}$ & $731-803$ \\
\hline Watzoyphius & CAC, MAQ, OTO, IN5, SRO, LAC, CON, PAL & $200-361$ \\
\hline Zelusia & SRO, CON, ORO & $227-695$ \\
\hline Caenis & CAC, MAQ, INF5, INF3, LAC, SRO, CSR, WSR & $200-308$ \\
\hline Coryphorus & CON & 248 \\
\hline Campylocia & SRO, LAC, CON, WSR, PMZ, ORO & $227-695$ \\
\hline Euthyplocia & $\mathrm{CHI}$ & 803 \\
\hline Amanahyphes & SRO, LAC, CON & $227-248$ \\
\hline Haplohyphes & YMG, $\mathrm{CHI}$ & $731-803$ \\
\hline Leptohyphes & ATI, CSR, WSR, ESR, PAL, LIM, LIA, YMG, CHI, CHA, TIO & $108-1912$ \\
\hline Lumahyphes & WSR & 308 \\
\hline Tricorythodes & ATI, CAC, SRO, CSR, WSR, ESR, PMZ, PMT, PAL, LIM, LIA, ORO, YMG, CHI & $108-803$ \\
\hline Tricorythopsis & PMZ & 321 \\
\hline Yaurina & ATI, CSR, WSR, ESR, PMZ, PMT, PAL, CHA & $180-1453$ \\
\hline Ecuaphlebia & ATI, SRO, LAC & $180-230$ \\
\hline Farrodes & ATI, IN3, IN5, SRO, LAC, CSR, WSR, ESR, PMZ, PAL, ORO, LIM, LIA, YMG & $180-731$ \\
\hline Fittkaulus & LAC, PMZ & $230-321$ \\
\hline Hagenulopsis & $\mathrm{ESR}, \mathrm{CHI}$ & $309-803$ \\
\hline Hydrosmilodon & PAL, CHI & $361-803$ \\
\hline Hylister & ATI & 180 \\
\hline Meridialaris & NIH, LUC, LUA, CHC, MAP, YUN, CAT1, PLQ, JOL, AJO, MAA & $2638-4411$ \\
\hline Miroculis & ATI, CAC, IN3, IN5, SRO, LAC, CSR, WSR, ESR, ORO & $180-695$ \\
\hline Simothraulopsis & ото & 222 \\
\hline Terpides & ATI, IN5, SRO, LAC, CSR, ESR, PMZ, YMG, CHI & $180-803$ \\
\hline Thraulodes & CSR, WSR, ESR, PAL, LIM, LIA, LAM, ORO, YMG, CHI, TIO & $308-1912$ \\
\hline Tikuna & IN5, LAC, CSR & $227-308$ \\
\hline Ulmeritoides & IN5, LAC & $227-230$ \\
\hline Homoeoneuria & WSR & 308 \\
\hline Lachlania & ATI & 180 \\
\hline Asthenopus & LAC & 230 \\
\hline Campsurus & SRO, ESR, PMZ & $227-321$ \\
\hline Hubbardipes & SRO & 227 \\
\hline
\end{tabular}



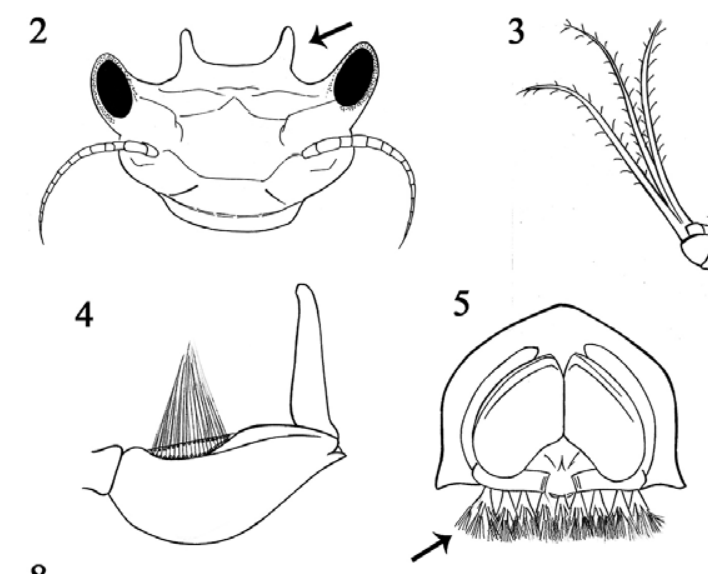

8
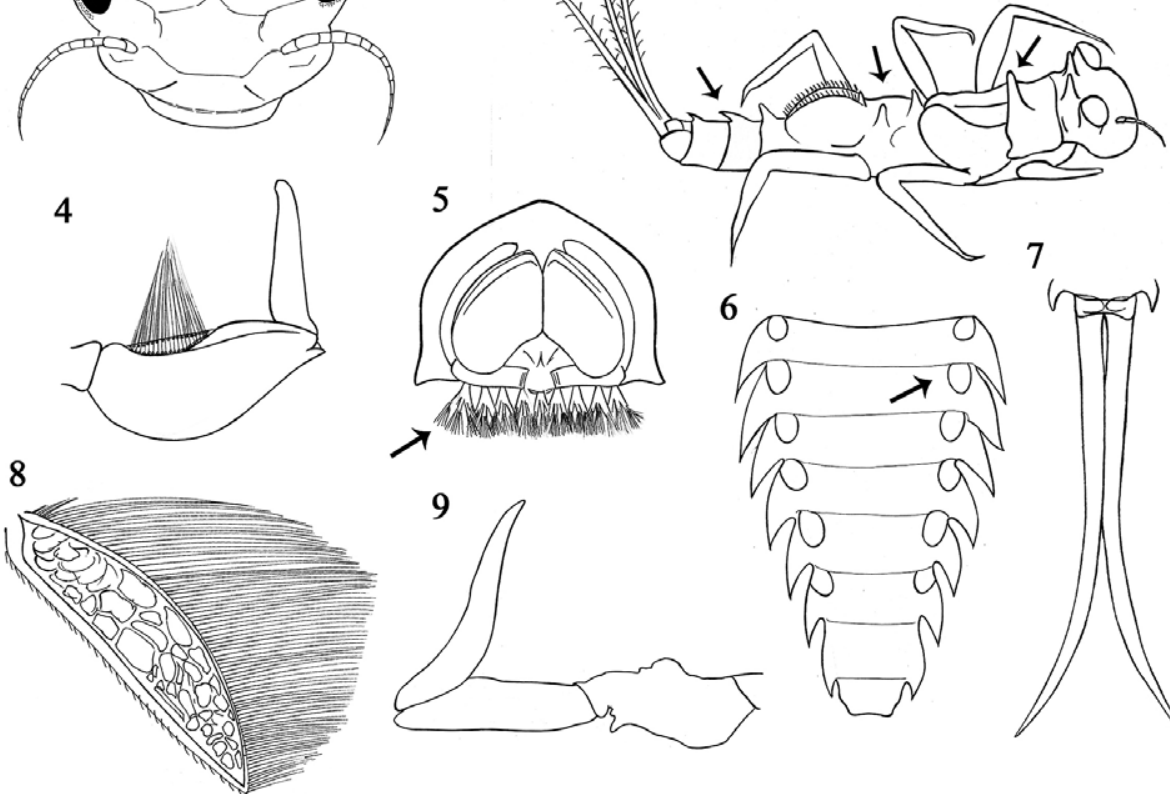

10

11

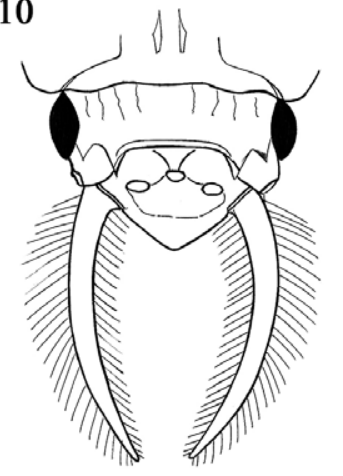

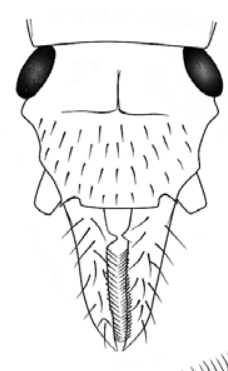

13

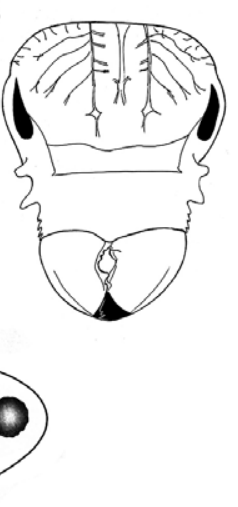

7

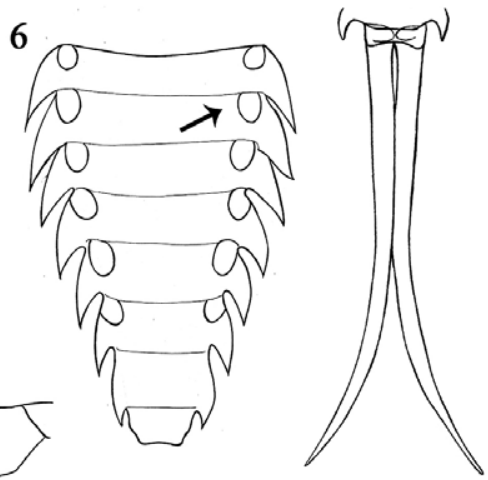

14

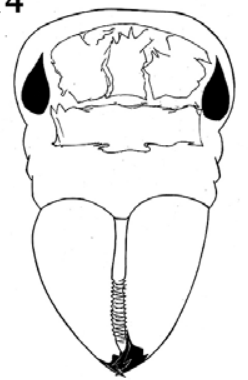

Figuras 2 - 14. Coryphoridae: 2. Vista frontal de la cabeza, Coryphorus. 3. Vista lateral de Coryphorus. Oligoneuriidae: 4. Fémur anterior, Lachlania. 5. Vista ventral de la cabeza, Lachlania. 6. Vista dorsal de los segmentos abdominales IIIX, Lachlania. 7. Vista dorsal de cercos, Lachlania. 8. Branquia laminar, Homoeoneuria. 9. Pata anterior, Homoeoneuria. Euthyplociidae: 10. Vista dorsal de cabeza, Campylocia. Polymitarcyidae: 11. Vista dorsal de cabeza, Campsurus. 12. Vista lateral de cabeza, Campsurus. 13. Vista dorsal de cabeza, Asthenopus. 14. Vista dorsal de cabeza, Hubbardipes.

8b. Colmillos mandibulares largos y delgados sin tubérculo sobre el margen medio (Fig. 14).

Hubbardipes Molineri, Salles \& Peters, 2015 (Fig. 105)

9a.Branquias abdominales operculares en el segmento II, cubriendo las restantes (Fig. 106-113).

9b.Branquias abdominales variables, pero las del segmento II nunca operculares.

10a.Branquias operculares del segmento II grandes subcuadrangulares, con dos costillas unidas en forma de "Y" (Fig. 17); branquia I filiforme (Fig. 18).

CAENIDAE

11

10b.Branquias operculares subcuadrangulares, triangulares, subtriangulares u ovales, nunca con dos costillas formando una "Y"; branquias laminares sin flecos; coxas variables, generalmente sin proyecciones. LEPTOHYPHIDAE (véase la clave de géneros)

11a.Branquias operculares con espinas cortas, fuertes y truncadas (Fig. 19); segmentos abdominales IV-VII con proyecciones posterolaterales largas y puntiagudas y largas que alcanzan la mitad del siguiente segmento (Fig. 20).
Brasilocaenis Puthz, 1975
11b.Branquias operculares sin espinas cortas, fuertes y truncadas; segmentos abdominales IV-VII con proyecciones posterolaterales largas que no alcanzan la mitad del siguiente segmento.

Caenis Stephens, 1835 (Fig. 106)

12a.Clípeo fusionado a la frente (Fig. 38 - 39), cabeza usualmente prognata (Fig. 37) (excepto en Terpides, Fittkaulus y Tikuna, que es hipognata); branquias abdominales compuestas de una lámina ventral y una dorsal (Fig. 41 - 42, Fig. 46, Fig. 49 - 50).

LEPTOPHLEBIIDAE (véase la clave de géneros)

12b.Clípeo no fusionado a la frente, cabeza hipognata; branquias en los segmentos abdominales I-V, I-VII, o II-VII con un sola lámina (Fig. 85 88). BAETIDAE (véase la clave de géneros)

\section{Familia Leptohyphidae \\ (Adaptado de Salles et al. 2018)}

1a. Branquias presentes en los segmentos abdominales II-V; branquias operculares subcuadradas con una línea transversal submedial (Fig. 24), que se tocan ligeramente en la zona media.

Amanahyphes Salles \& Molineri, 2006 (Fig. 107) 


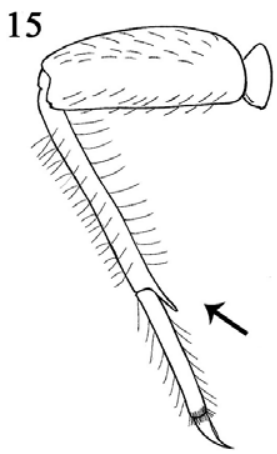

18

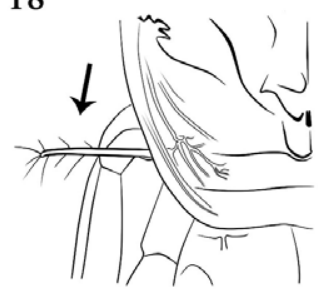

21

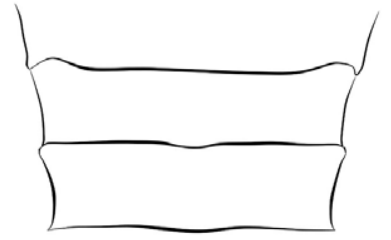

16

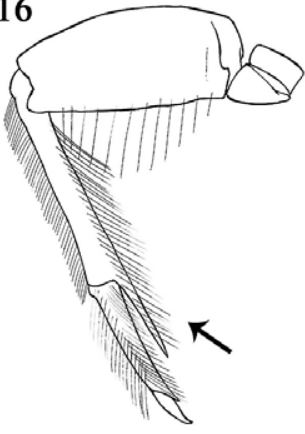

19
17

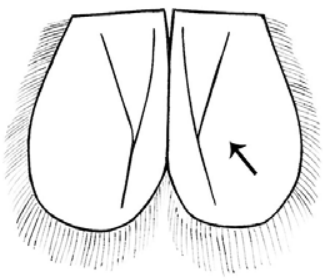

20

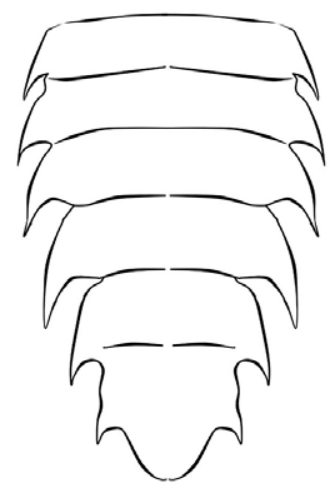

22

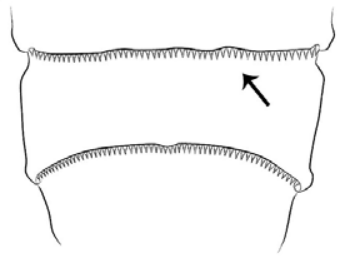

26

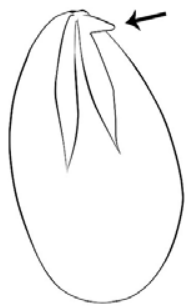

23

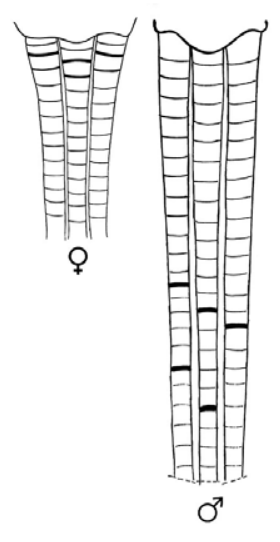

Figuras 15 - 26. Euthyplociidae: 15. Pata anterior, Campylocia. 16. Pata anterior, Euthyplocia. Caenidae: 17. Branquias operculares, Caenis. 18. Branquia I, Caenis. 19. Branquia opercular, Brasilocaenis (tomado de Lima et al. 2019). 20. Vista ventral de los segmentos abdominales del IV-IX, Brasilocaenis (tomado de Lima et al. 2019). Leptohyphidae: 21. Vista dorsal de los segmentos abdominales del I-V, Haplohyphes. 22. Vista dorsal de los segmentos abdominales del I-IX, Leptohyphes. 23. Filamentos caudales, Leptohyphes (hembra y macho). 24. Branquia opercular, Amanahyphes. 25. Branquia opercular, Tricorythopsis. 26. Branquia opercular, Leptohyphes.

1b. Branquias presentes en los segmentos abdominales II-VI; branquias operculares de forma variable con o sin línea transversal submedial, nunca se tocan en la zona media. 2

2a. Tergo abdominal I-V sin espinas en el margen posterior (Fig. 21). 3

2b. Tergo abdominal I-IX con espinas pequeñas en el margen posterior (Fig. 22).

4

3a. Base del abdomen ligeramente más amplia que el ápice (Fig. 108); margen anterior del fémur de la pata anterior con espinas pequeñas pero robustas (Fig. 27).

Haplohyphes Allen, 1966 (Fig. 108)

3b. Base del abdomen mucho más amplia que el ápice (Fig. 109); margen anterior del fémur de la pata anterior sin espinas (Fig. 28).

Tricorythodes Ulmer, 1920 (Fig. 109)

4a. Branquias operculares ovaladas con una línea transversa clara en la zona media o apical (excepto T. rondoniensis y T. spongicola) (Fig. 25).
Tricorythopsis Traver 1958 (Fig. 110) 4b. Branquias operculares ovaladas o subovaladas pero nunca con una línea transversa clara en la zona media o apical.

5a. Fémures de la pata media y posterior con una hilera transversal de espinas en la base (Fig. 29), y generalmente con una quilla mediolongitudinal; branquias operculares con espina basal (Fig. 26); anulaciones oscuras en los filamentos caudales, en la base de los filamentos (hembras) o a la mitad de éstos (machos) (Fig. 23).

Leptohyphes Eaton, 1882 (Fig. 111)

5b. Fémures de la pata media y posterior sin una hilera transversal de espinas en la base, ni una quilla longitudinal; branquias operculares sin espina basal; filamentos caudales sin anulaciones oscuras.

6a. Palpo maxilar biarticulado (Fig. 31).

7

6b. Palpo maxilar triarticulado (Fig. 32).

8

7a. Palpo maxilar generalmente con seta apical (Fig. 33); uña tarsal anterior con una hilera subapical de 4 dentículos (Fig. 34).

Allenhyphes Hofmann \& Sartori, 1999 (en parte) 

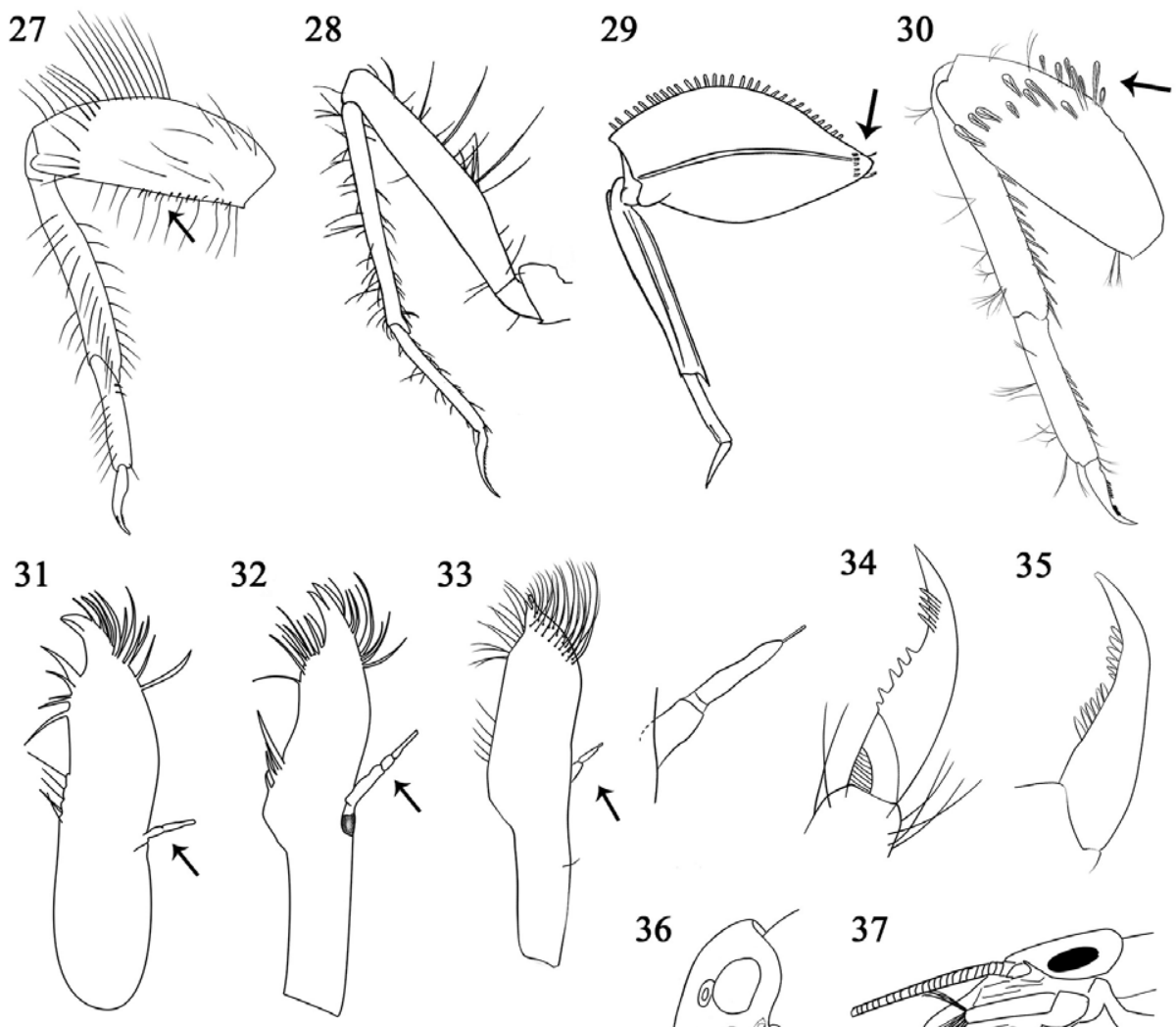

33

38

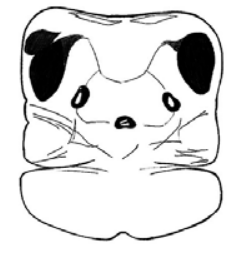

39

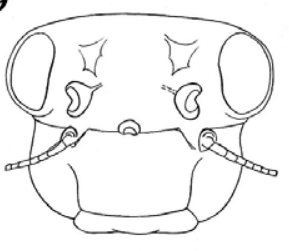

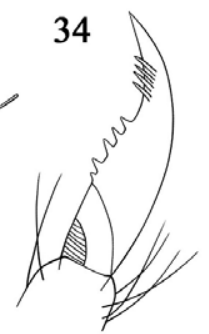

35

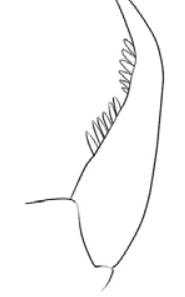

36

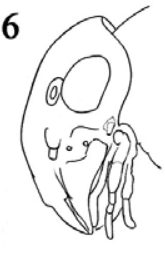

37

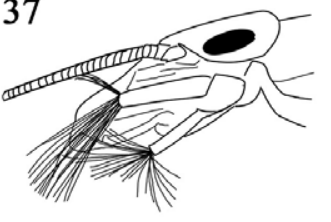

40

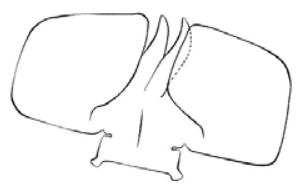

Figuras 27 - 40. Leptohyphidae: 27. Pata anterior, Haplohyphes. 28. Pata anterior, Tricorythodes. 29. Pata posterior, Leptohyphes. 30. Pata anterior, Lumahyphes. 31. Palpo maxilar, Yaurina. 32. Palpo maxilar, Lumahyphes. 33. Palpo maxilar, Allenhyphes (tomado de Salles et al. 2018). 34. Uña tarsal, Allenhyphes (tomado de Allen 1973). 35. Uña tarsal anterior, Yaurina. Leptophlebiidae: 36. Vista lateral de cabeza hipognata, Terpides. 37. Vista lateral de cabeza prognata, Hydrosmilodon. 38. Vista dorsal de cabeza, Hydrosmilodon. 39. Vista dorsal de cabeza, Meridialaris. 40. Vista dorsal de la glosa, Miroculis.

7b. Palpo maxilar generalmente sin seta apical; uña tarsal anterior con una hilera subapical de 6-7 dentículos (Fig. 35).

Yaurina Molineri, 2001 (Fig. 112)

8a.Dorso de los fémures anteriores con setas espatuladas bífidas o aserradas (Fig. 30). Lumahyphes Molineri, 2004 (Fig. 113)

8b. Dorso de los fémures anteriores con setas espatuladas no bífidas ni aserradas.

Allenhyphes Hofmann \& Sartori, 1999 (en parte)

Familia Leptophlebiidae

(Adaptada de Domínguez et al. 2006, Domínguez y

Fernández 2009)

1a.Cuerpo subcilíndrico; cabeza hipognata (Fig. 36); filamentos caudales con setas largas en los márgenes internos de los cercos y márgenes laterales del filamento terminal (Fig. 51).

1b. Cuerpo aplanado; cabeza prognata (Fig. 37); filamentos caudales con espinas o setas pequeñas pero no en la disposición de arriba.
2a.Branquias que se afinan gradualmente hacia el ápice y terminan en un único filamento (Fig. 41); uña tarsal con dentículo medial grande (Fig. 56). Terpides Demoulin, 1966 (Fig. 114)

2b. Lámina dorsal de las branquias II-VI terminadas en un filamento y 1 ó 2 lóbulos laterales; uña tarsal sin dentículo medial grande.

3a.Lámina ventral de las branquias II-VI (Fig. 42) con lóbulos no desarrollados. Fittkaulus Savage \& Peters, 1978 (Fig. 115)

3b. Lámina ventral de las branquias II-VI con un lóbulo apical interno y otro externo más desarrollado (Fig. 43).

$$
\text { Tikuna Savage, Flowers \& Porras, } 2005 \text { (Fig. 116) }
$$

4a. Ancho del labro igual que el ancho de la cabeza (Fig. 38).

4b.Ancho del labro menor que el ancho de la cabeza (Fig. 39).

5a. Branquias con seis o más proyecciones digitiformes (Fig. 44 - 45).

5b.Branquias terminan en un único proceso digitiforme (Fig. 46). 

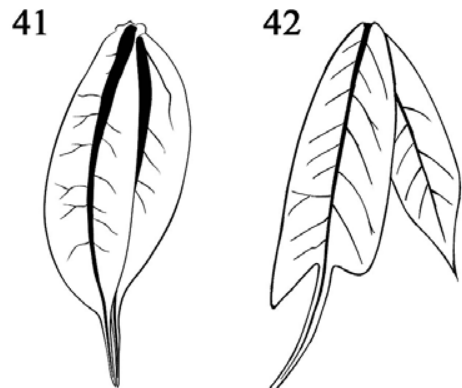

46

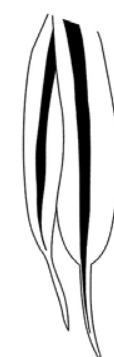

47

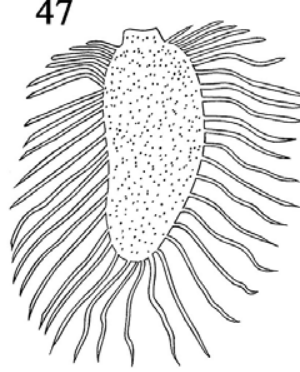

52

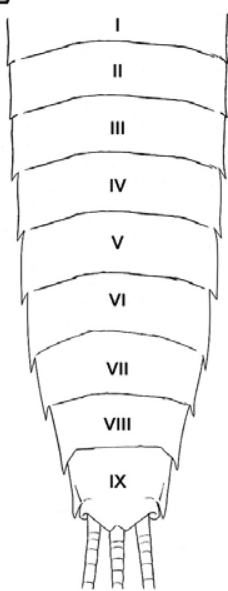

43

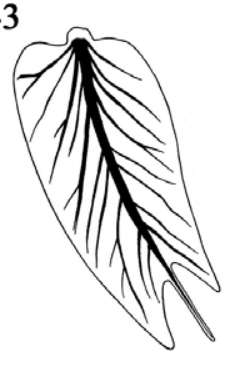

48

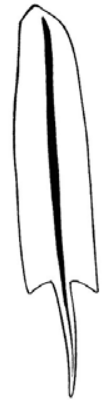

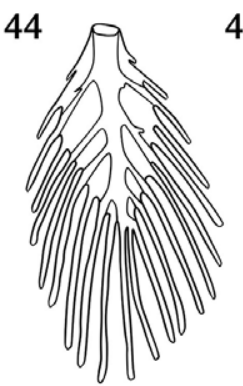

50

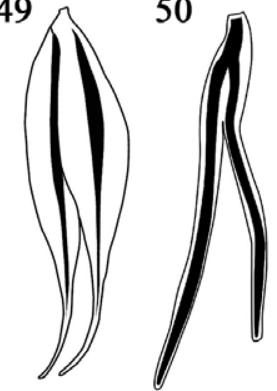

54

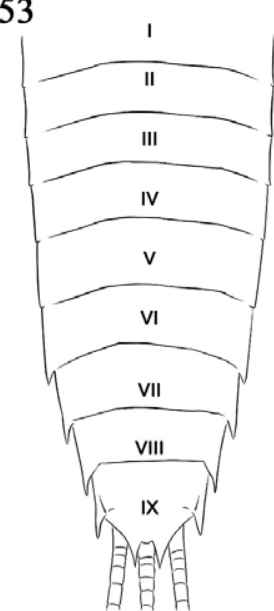

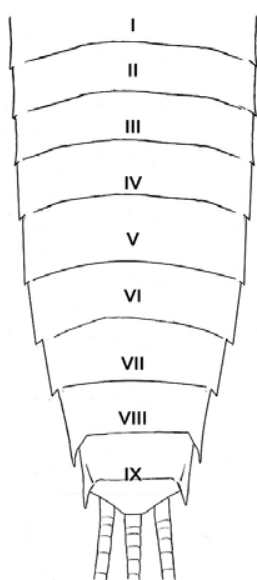

45

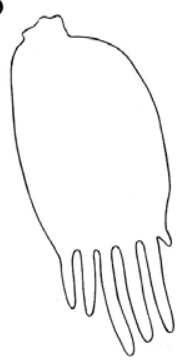

51

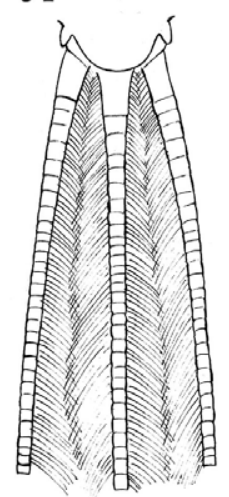

55

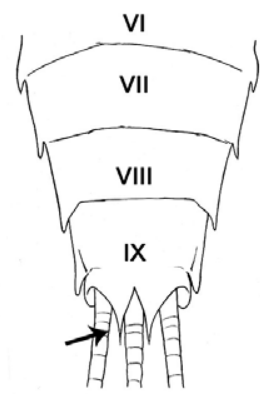

Figuras 41 - 55. Leptophlebiidae: 41. Branquia laminar, Terpides. 42. Branquia laminar, Fittkaulus. 43. Branquia laminar ventral, Tikuna. 44. Branquia, Traverella (tomado de Edmunds 1948). 45. Branquia laminar, Hylister. 46. Branquia laminar, Hydrosmilodon. 47. Branquia laminar, Ulmeritoides. 48. Branquia laminar, Miroculis. 49. Branquia laminar, Simothraulopsis. 50. Branquia laminar, Farrodes. 51. Filamentos caudales, Terpides. 52. Vista ventral de los segmentos abdominales, Thraulodes. 53. Vista ventral de los segmentos abdominales, Ecuaphlebia. 54. Vista ventral de los segmentos abdominales, Farrodes. 55. Vista ventral de los últimos cuatro segmentos abdominales, Hagenulopsis.

6a. Branquia oval con flecos en su márgenes (Fig. 44); uñas tarsales con dentículos subiguales (Fig. 57).

Traverella Edmunds, 1948

6b. Branquias rectangulares truncadas apicalmente con 4 a 10 proyecciones apicales filamentosas (Fig. 45); uñas tarsales con dentículo apical más grande que los demás (Fig. 58).

Hylister Domínguez \& Flowers, 1989 (Fig. 117)

7a. Colmillo maxilar aproximadamente de un cuarto o menos del ancho del ápice de la maxila (Fig. 61).

Paramaka Savage \& Domínguez, 1992

7b. Colmillo maxilar aproximadamente de la mitad o más del ancho del ápice de la maxila (Fig. 62).

Hydrosmilodon Flowers \& Domínguez, 1992 (Fig. 118)

8a. Margen de las branquias con procesos filamentosos (Fig. 47); borde anteromedial del labro con diente grande (Fig. 63).

Ulmeritoides Traver, 1959 (Fig. 119) 9a. Branquias con un largo y delgado filamento terminal de aproximadamente la mitad de longitud de la parte basal, parte basal usualmente con lóbulos laterales (Fig. 48); glosas curvadas ventralmente (Fig.40).

Miroculis Edmunds, 1963 (Fig. 120)

9b. Branquias lanceoladas anchas o delgadas, si el filamento distal de la branquia está presente constituye un tercio o menos de la longitud de la porción basal; glosas no curvadas ventralmente. $\mathbf{1 0}$

10a.Márgenes laterales del clípeo divergentes apicalmente (Fig. 64); proyecciones posterolaterales presentes en los segmentos abdominales II o III al IX (Fig. 52).

11

10b.Márgenes laterales del clípeo paralelos apicalmente (Fig. 65); proyecciones posterolaterales presentes en los segmentos abdominales VI o VII o VIII al IX (Fig. 53 - 54).

12

11a. Márgenes laterales del labro aguzados (Fig. 64); margen externo de las mandíbulas sin un penacho de setas (Fig. 67).

Thraulodes Ulmer, 1920 (Fig. 121)

8b. Márgenes de las branquias sin procesos filamentosos o con un filamento terminal. 

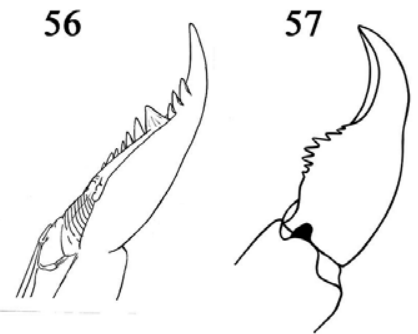

58
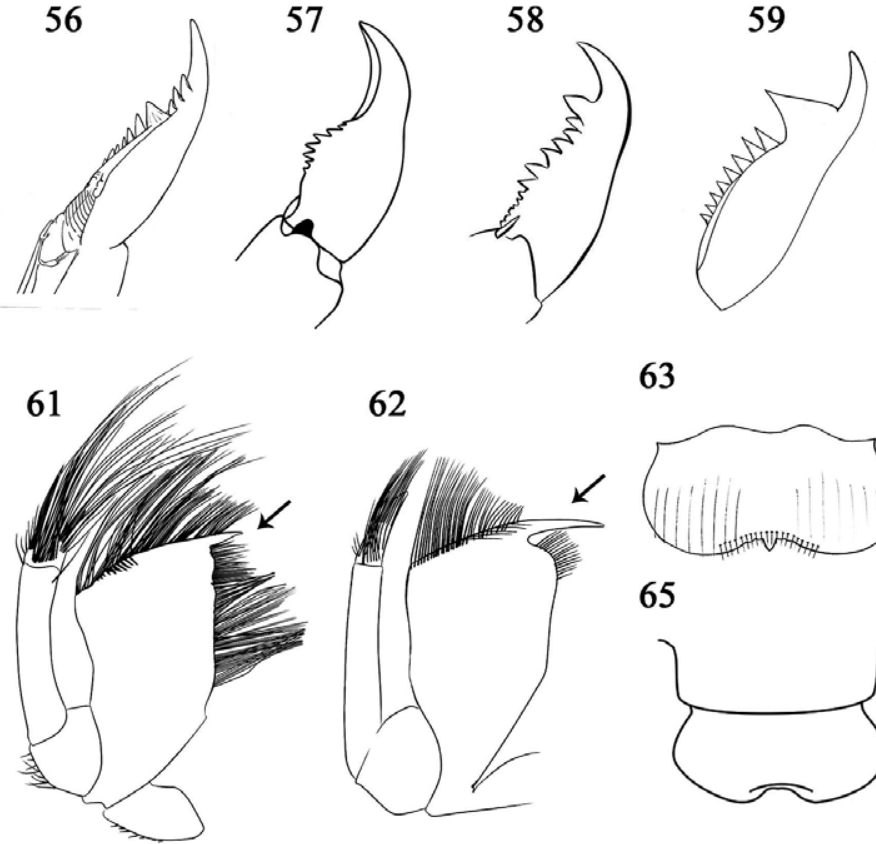

62
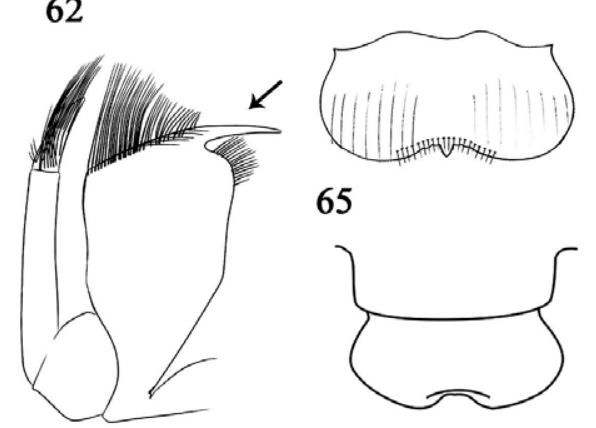

65
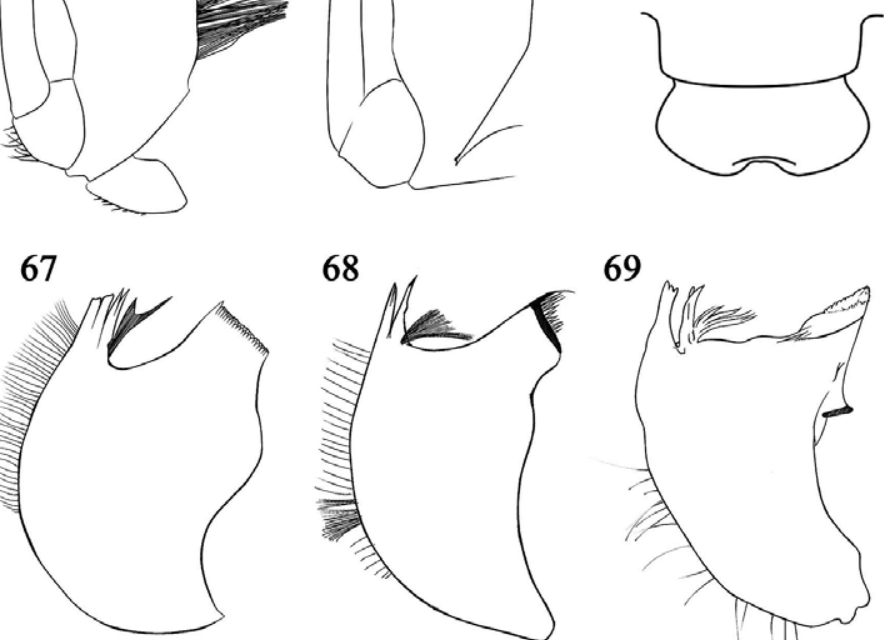

68

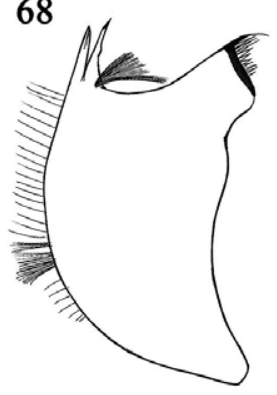

69

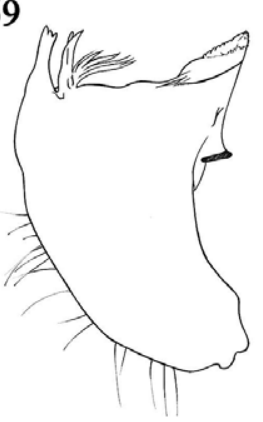

66

60

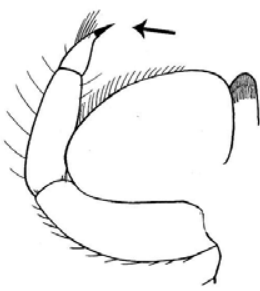

64
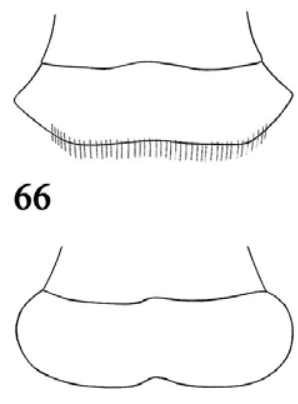

70

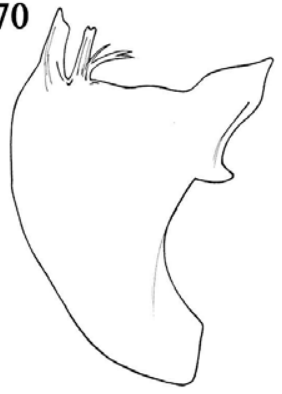

Figuras 56 - 70. Leptophlebiidae: 56. Uña tarsal, Terpides. 57. Uña tarsal, Traverella (tomado de Edmunds 1948). 58. Uña tarsal, Hylister (adaptado de Kluge 2007). 59. Uña tarsal, Simothraulopsis. 60. Palpo labial, Hagenulopsis. 61. Colmillo maxilar, Paramaka (Tomado de Mariano 2011) 62. Colmillo maxilar, Hydrosmilodon. 63. Labro, Ulmeritoides. 64. Clípeo y labro, Thraulodes. 65. Clípeo y labro, Hagenulopsis. 66. Clípeo y labro, Meridialaris.67. Mandíbula izquierda, Thraulodes. 68. Mandíbula izquierda, Meridialaris. 69. Mandíbula derecha, Ecuaphlebia. 70. Mandíbula derecha, Farrodes.

11b.Márgenes laterales del labro redondeados (Fig. 66); margen externo de las mandíbulas con un penacho de setas (Fig. 68).

Meridialaris Peters \& Edmunds, 1972 (Fig. 122)

12a.Branquias lanceoladas con ancho aproximadamente 1/5 de su longitud (Fig. 49); dentículo subapical de la uña tarsal notoriamente más grande que los otros (Fig. 59).

\section{Simothraulopsis Demoulin, 1966 (Fig. 123)}

12b.Branquias delgadas con ancho aproximadamente $1 / 10$ de su longitud (Fig. 50).

13a.Margen posterior del esterno IX con proyecciones agudas (Fig. 55); ápice del segmento III del palpo labial esclerotizado (Fig. 60).

$$
\text { Hagenulopsis Ulmer, } 1920 \text { (Fig. 124) }
$$

13b. Margen posterior del esterno IX sin proyecciones agudas; segmento III del palpo labial no esclerotizado.

14a.Mandíbula con setas en la mitad basal del margen externo (Fig. 69); proyecciones posterolaterales en los segmentos abdominales VI al IX incrementándose gradualmente en tamaño (Fig. 53). Ecuaphlebia Domínguez, 1988 (Fig. 125)

14b.Mandíbula sin setas en el margen externo (Fig. 70); proyecciones posterolaterales de los segmentos abdominales VIII y IX mucho más desarrolladas que en los otros segmentos (Fig. 54).
Familia Baetidae

(Adaptada de Domínguez et al. 2006, Domínguez y Fernández 2009)

1a. Uñas tarsales espatuladas (Fig. 77), perpendiculares al tarso. Camelobaetidius Demoulin, 1966 (Fig. 127)

1b.Uñas tarsales aguzadas en el mismo eje longitudinal de la pata, con o sin dentículos (Fig. 78 - 83).

2a.Branquias presentes en los segmentos I - V, ventrolaterales (Fig. 71). Baetodes Needham \& Murphy, 1924 (Fig. 128)

2b. Branquias presentes en los segmentos I - VII, dorsolaterales (Fig. 72 - 73).

3

3a. Branquias abdominales con expansiones plegadas en la base (Fig. 85); uñas tarsales anteriores con 2 hileras de dentículos cilíndricos (Fig. 78).

Callibaetis Eaton, 1881 (Fig. 129)

3b. Branquias abdominales simples; uñas tarsales anteriores con dentículos nunca cilíndricos.

4

4a. Uñas tarsales sin dentículos o muy pequeños (Fig. 79); tibia con arco de setas justo debajo de la articulación con el fémur (Fig. 76). Cloeodes Traver, 1938 (Fig. 130)

Farrodes Peters, 1971 (Fig. 126) 
71

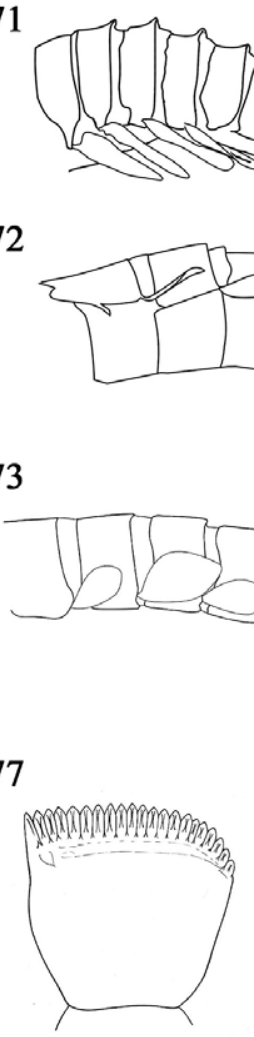

74
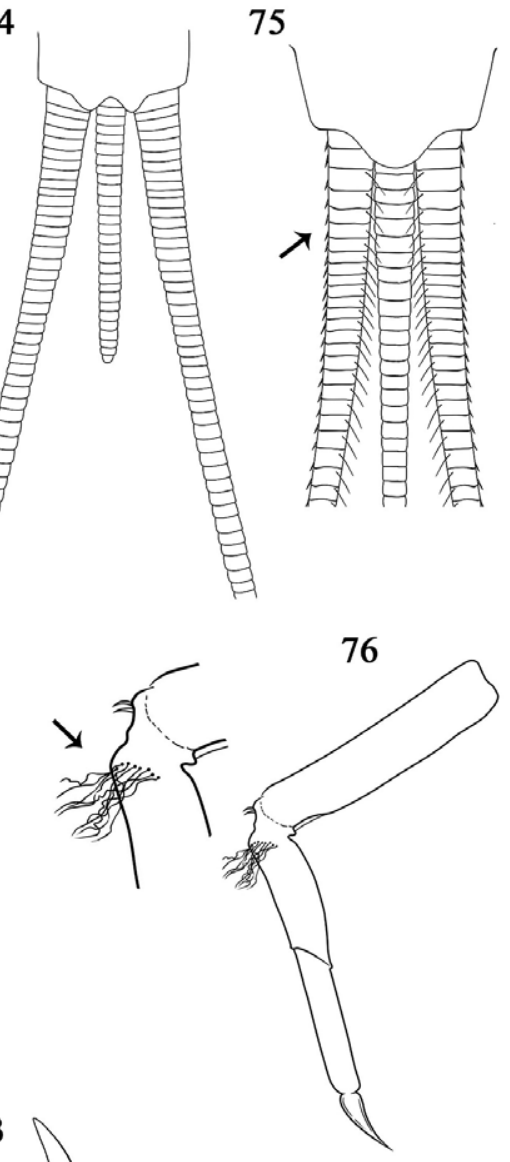

84

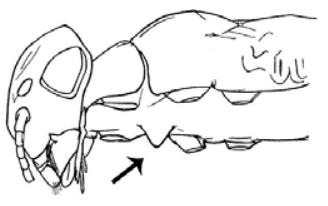

Figuras 71 - 84. Baetidae: 71. Segmentos abdominales, Baetodes. 72. Segmentos abdominales, Andesiops. 73. Segmentos abdominales, Nanomis. 74. Filamentos caudales, Prebaetodes. 75. Filamentos caudales, Mayobaetis. 76. Tibia anterior, Cloeodes. 77. Uña tarsal espatulada, Camelobaetidius. 78. Uña tarsal, Callibaetis. 79. Uña tarsal, Cloeodes. 80. Uña tarsal, Mayobaetis. 81. Uña tarsal, Nanomis. 82. Uña tarsal, Andesiops. 83. Uña tarsal, Guajirolus (tomado de Salles et al. 2018). 84. Mesosternón, Waltzoyphius.

4b. Uñas tarsales con dentículos; tibia sin arco de setas justo debajo de la articulación con el fémur.

5a. Filamento terminal menor a la mitad de los cercos (Fig. 74); uña tarsal con una fuerte seta subapical (Fig. 80).

6

5b. Filamento terminal mayor a la mitad de los cercos; uña tarsal con o sin seta, si está presente es débil.

7

6a. Escapo y pedicelo dorsoventralmente aplanado, longitud del escapo tres veces más largo que el pedicelo (Fig. 88); cercos con espinas en el margen lateral (Fig. 75).

Mayobaetis Waltz \& McCafferty, 1985 (Fig. 131)

6b. Escapo y pedicelo cilíndricos o subcilíndricos, longitud del escapo subigual al pedicelo (Fig. 90); cercos sin espinas en el margen lateral.

Prebaetodes Lugo-Ortiz \& McCafferty, 1996 (Fig. 132)

7a. Branquia I presente (en caso se haya desprendido, observar una zona esclerotizada en el lugar de donde ésta emerge).

7b. Branquia I ausente (no se observa una zona esclerotizada en lugar donde suelen emerger las branquias). 8a. Uña tarsal con una hilera de dentículos (Fig. 81); branquias con borde no completamente esclerotizado (Fig. 86). Nanomis Lugo-Ortiz \& McCafferty, 1999 (Fig. 133)

8b. Uña tarsal con dos hileras de dentículos (Fig. 81); branquias con borde completamente esclerotizado. 9

9a. Protuberancia en el mesoesterno presente (Fig. 84).

Waltzoyphius McCafferty \& Lugo-Ortiz, 1995 (Fig. 134)

9b. Protuberancia en el mesoesterno ausente.

10

10a.Uña tarsal con dos setas débiles subapicales (Fig. 82); branquias sin tráqueas, si están presentes, muy débiles).

Andesiops Lugo-Ortiz \& McCafferty, 1999 (Fig. 135)

10b.Uña tarsal sin setas; branquias con tráqueas bien definidas (Fig. 87 - 88).

11

11a.Mandíbula derecha con incisivos divididos desde su base (Fig. 94); prosteca delgada y bífida, sin seta transversal (Fig. 94).

Paracloeodes Day, 1955 


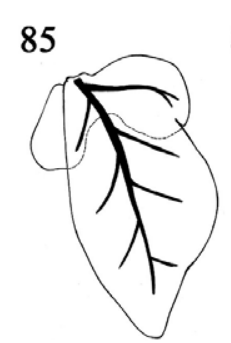

91

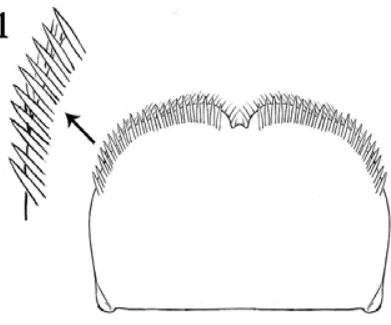

87

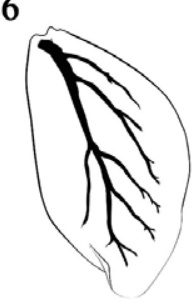

92
88

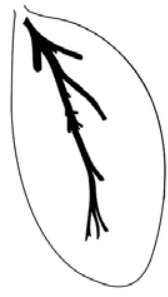

92
89

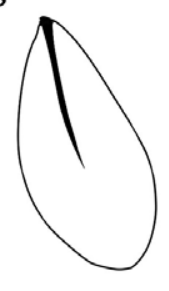

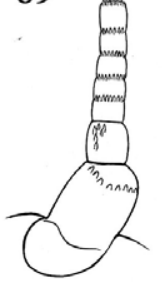

90

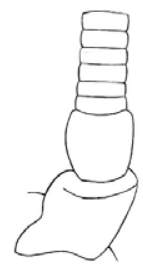

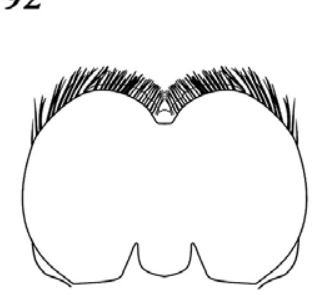

93

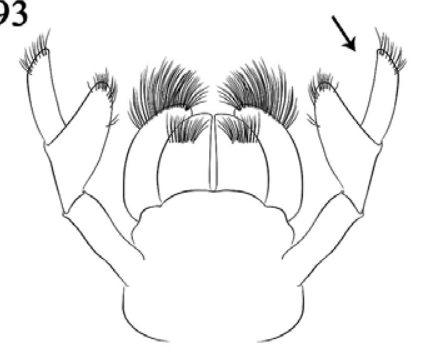

97

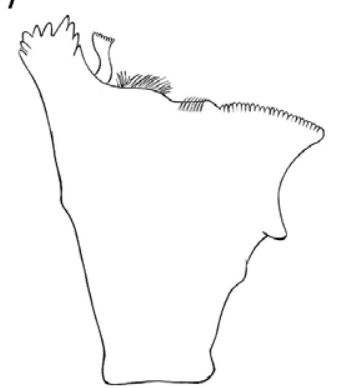

Figuras 85 - 97. Baetidae: 85. Branquias abdominales plegadas en la base, Callibaetis. 86. Branquia abdominal, Nanomis. 87. Branquia abdominal, Americabaetis. 88. Branquia abdominal, Zelusia. 89. Antena, Mayobaetis. 90. Antena, Prebaetodes. 91. Labro, Aturbina. 92. Labro, Cryptonympha. 93. Palpo labial largo, Guajirolus (tomado de Salles et al. 2018). 94. Mandíbula derecha, Paracloeodes (Tomado de Nieto \& Salles 2006) 95. Mandíbula derecha, Aturbina. 96. Mandíbula derecha, Cryptonympha. 97. Mandíbula derecha, Americabaetis.

11b.Mandíbula derecha con incisivos fusionados (Fig. 95 - 96); prosteca robusta y con seta transversal (Fig. 95 - 96).

12

12a.Mandíbula derecha con seta transversal en la parte basal de la prosteca (Fig. 95); labro con setas submarginales aplanadas terminando en punta (Fig. 91).

Aturbina Lugo-Ortiz \& McCafferty, 1996 (Fig. 136)

12b.Mandíbula derecha con seta transversal en la parte media de la prosteca (Fig. 96); labro con borde submarginal con setas simples (Fig. 92).

Cryptonympha Lugo-Ortiz \& McCafferty, 1998 (Fig. 137)

13a.Segmento distal del palpo labial corto (tan largo como ancho); uña tarsal con dentículo distal ligeramente largo o subigual en tamaño a los demás dentículos.

13b.Segmento distal del palpo labial largo (tres veces más largo que ancho) (Fig. 93); uña tarsal con dentículo distal mucho más largo que los demás dentículos (Fig. 83).

Guajirolus Flowers, 1985 (Fig. 138)

14a.Setas entre el incisivo y la mola de las mandíbulas (Fig. 97); branquias con tráquea ramificada (Fig. 87).

Americabaetis Kluge, 1992 (Fig. 139)

14b.Ausencia de setas entre el incisivo y la mola de las mandíbulas; branquias con tráquea no ramificada (Fig. 88).

Zelusia Lugo-Ortiz \& McCafferty, 1998 (Fig. 140)

\section{Discusión}

Los efemerópteros peruanos hasta la década de 1960 fueron poco estudiados en aspectos, taxonómicos (Hubbard 1982), biológicos y ecológicos. Sin embargo, a comienzos de la década de los 80s hubo un incremento en su estudio, que se ajusta al desarrollo de la limnología en el mundo (Melo et al. 2006) y a los trabajos científicos realizados en el Neotrópico (Padial et al. 2008). La clave presentada constituye un nuevo aporte para el conocimiento de este orden en el sudeste del Perú. Se incluyen 48 géneros de un amplio gradiente altitudinal, considerados similares a los reportados en estratos altitudinales bajos y altos de otras quebradas de América del Sur. Tal es el caso de los géneros de las familias Baetidae y Leptophlebiidae que fueron recolectadas en los Andes de Colombia (Gutiérrez \& Dias 2015), Ecuador (Jacobsen 2008, Anderson et al. 2011) y Bolivia (Jacobsen \& Marín 2008, Molina et al. 2008). La mayoría de los géneros colectados habitan en cuerpos de agua permanentes y de corriente moderada, donde es común encontrar la mayor diversidad del orden (Barber-James et al. 2008, Brittain 


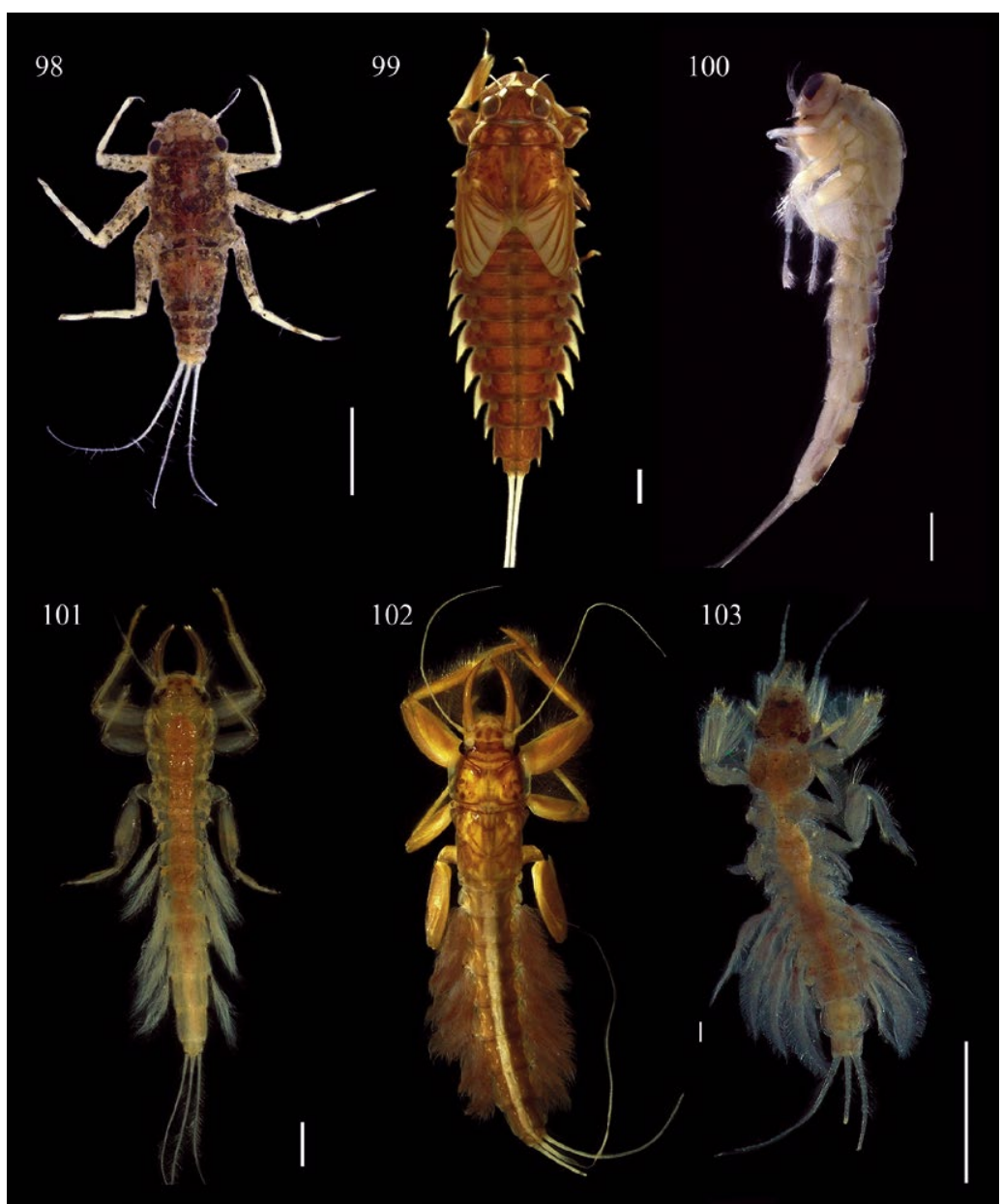

Figuras 98-103. Coryphoridae: 98. Coryphorus. Oligoneuriidae: 99. Lachlania. 100. Homoeoneuria. Euthyplociidae: 101. Campylocia. 102. Euthyplocia. Polymitarcyidae: 103. Campsurus. (Escala $=1 \mathrm{~mm}$ ).

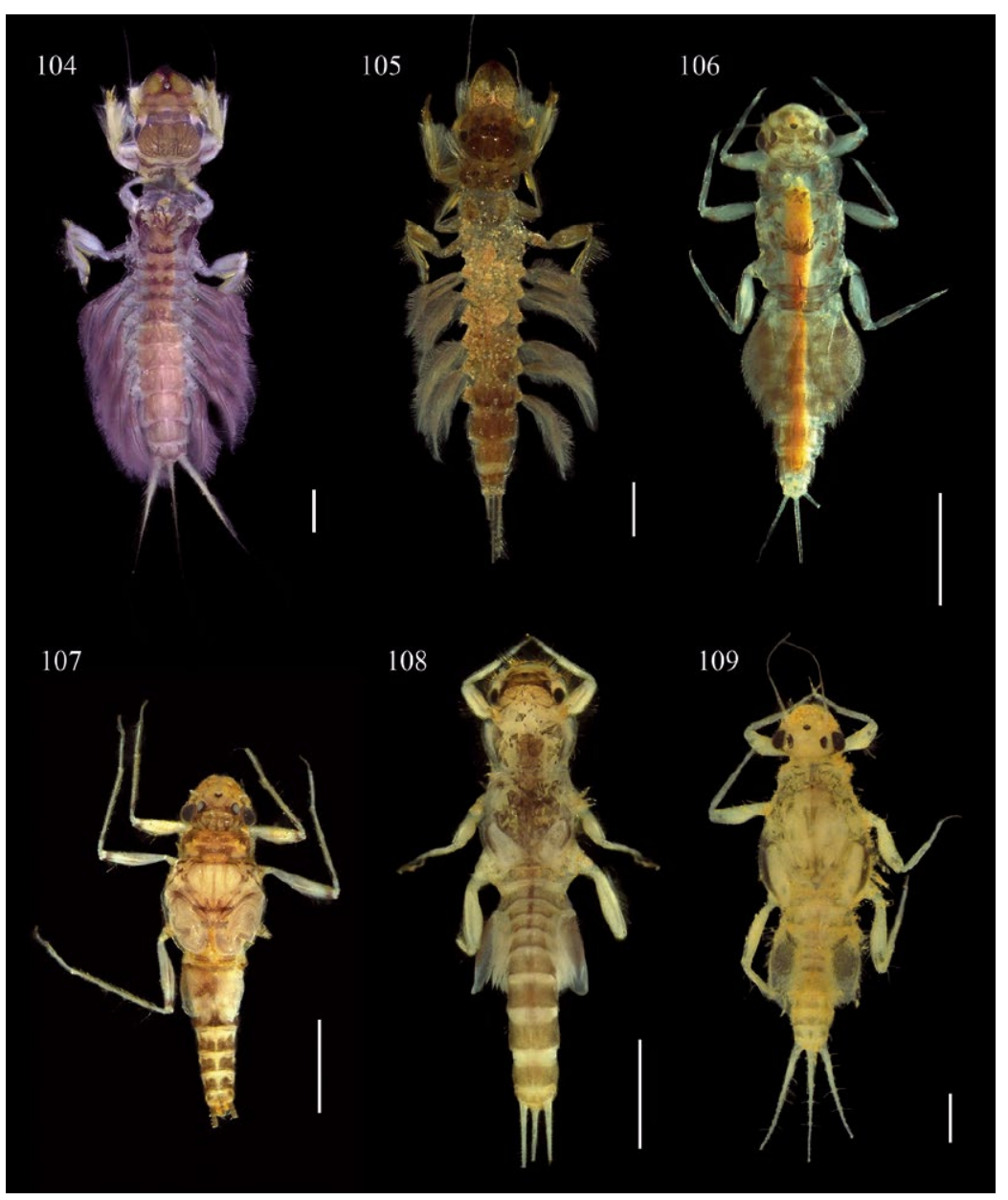

Figuras 104 - 109. Polymitarcyidae: 104. Asthenopus. 105. Hubbardipes. Caenidae: 106. Caenis. Leptohyphidae: 107. Amanahyphes. 108. Haplohyphes. 109. Tricorythodes. (Escala= $1 \mathrm{~mm}$ ). 

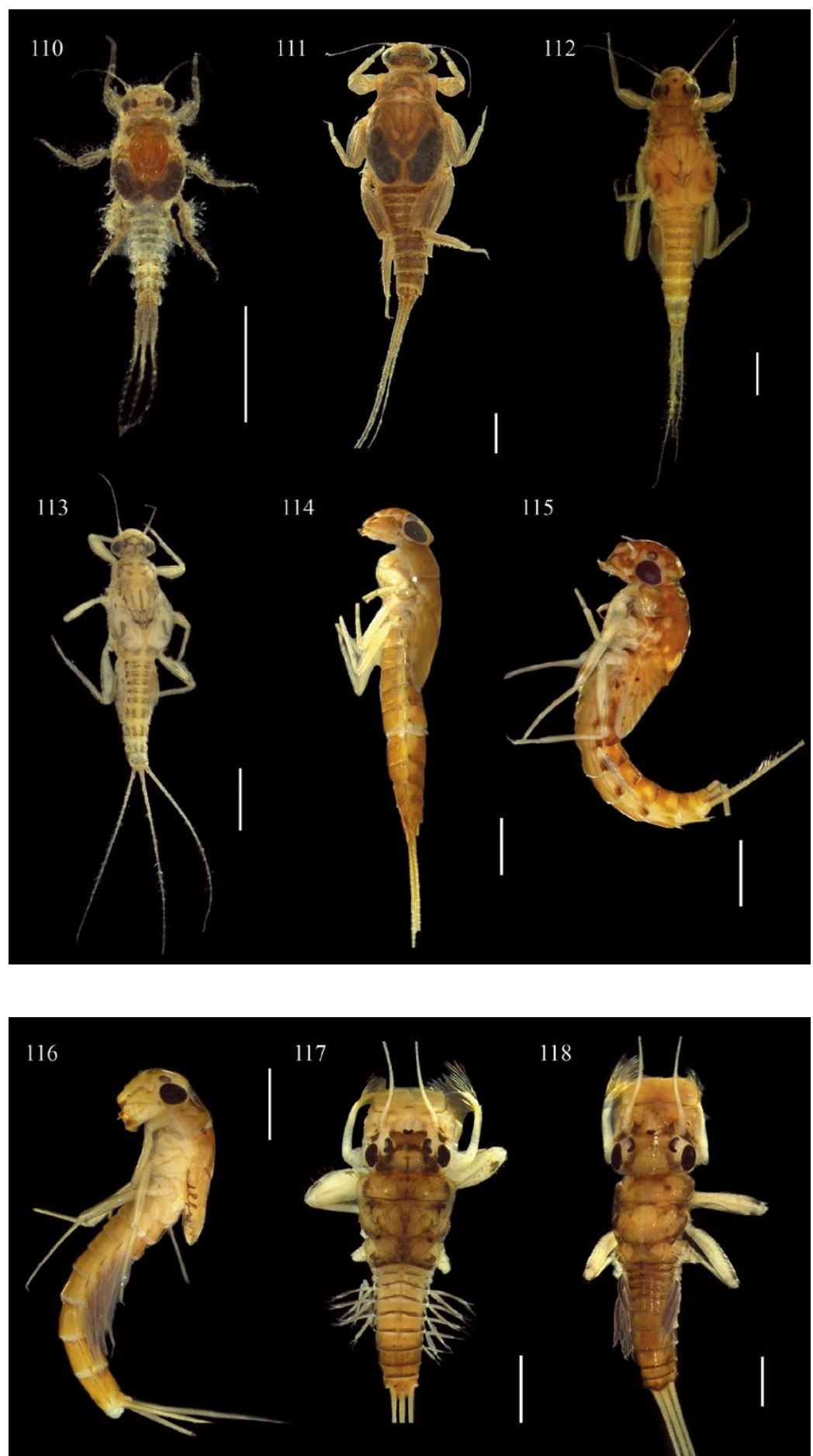

118

119

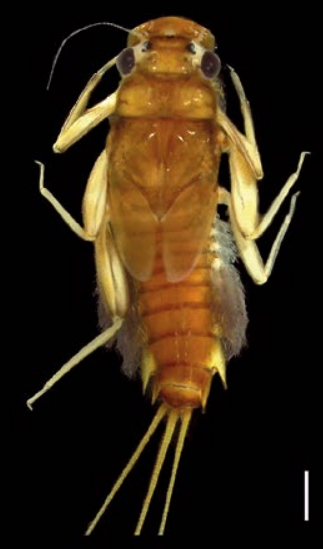

120

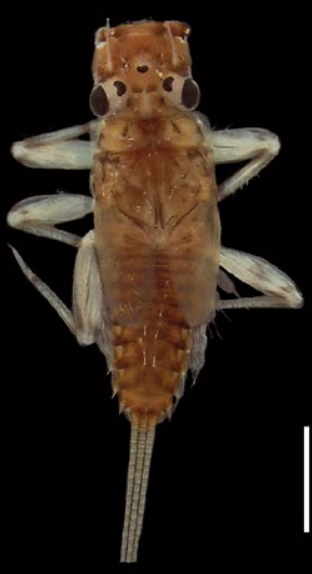

Figuras 110 - 115. Leptohyphidae: 110. Tricorythopsis. 111. Leptohyphes. 112. Yaurina. 113. Lumahyphes. Leptophlebiidae: 114. Terpides. 115. Fittkaulus. (Escala= $1 \mathrm{~mm})$.
Figuras 116 - 121. Leptophlebiidae: 116 . Tikuna. 117. Hylister. 118. Hydrosmilodon. 119. Ulmeritoides. 120. Miroculis. 121. Thraulodes. (Escala $=1 \mathrm{~mm})$. 


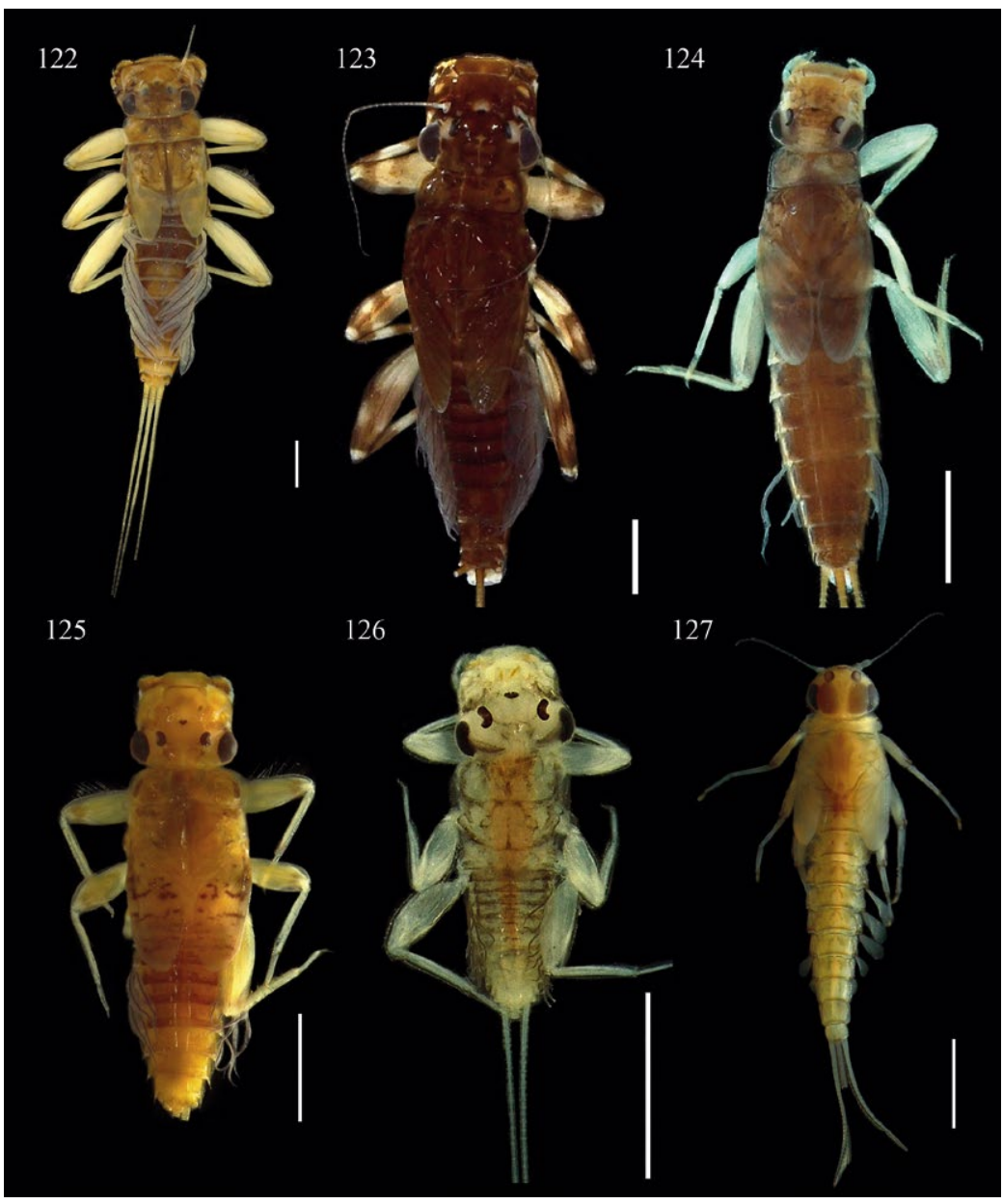

Figuras 122 - 127. Leptophlebiidae: 122. Meridialaris. 123. Simothraulopsis. 124. Hagenulopsis. 125. Ecuaphlebia. 126. Farrodes. Baetidae: 127. Camelobaetidius. (Escala $=1$ $\mathrm{mm})$.

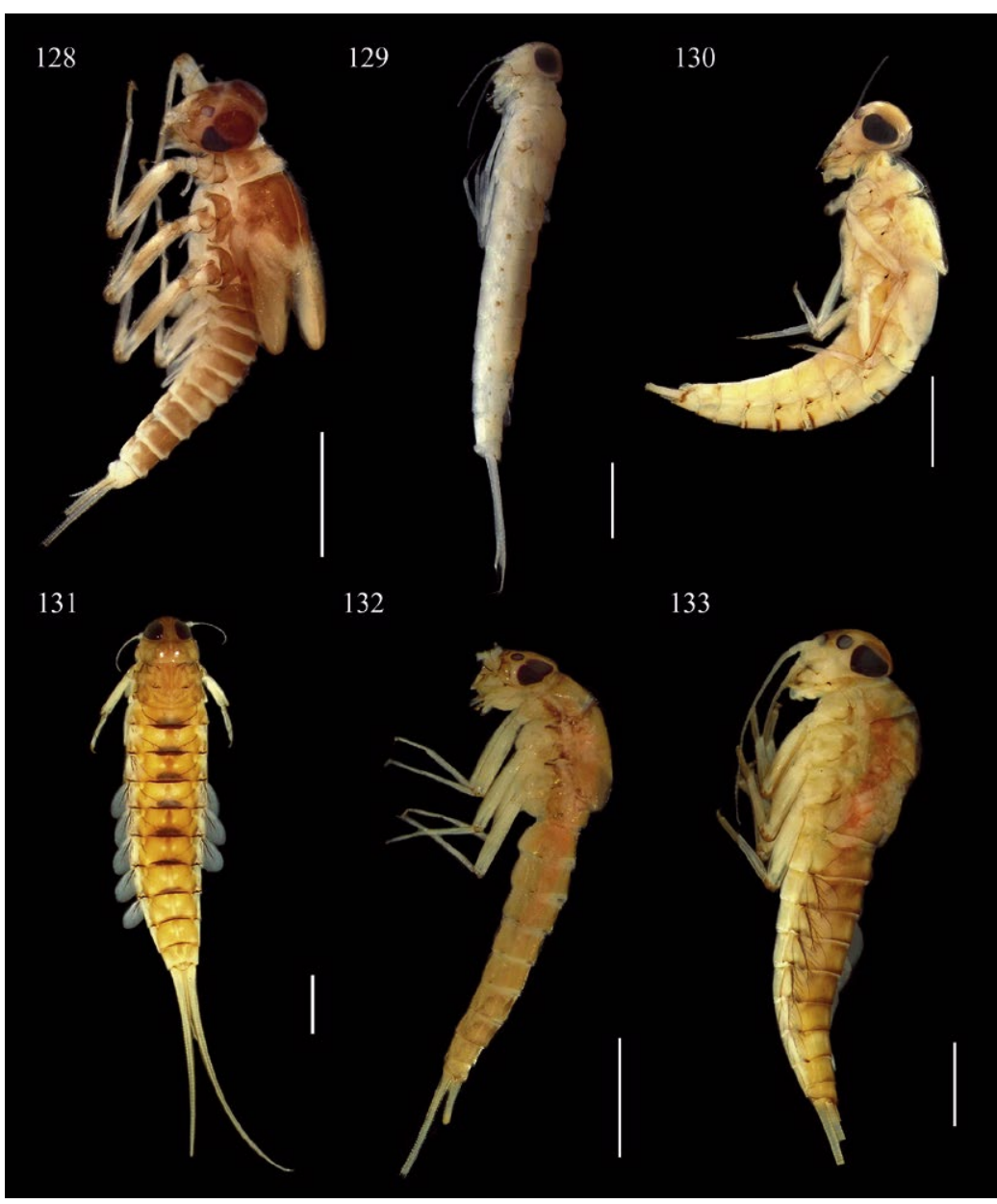

Figuras 128 - 133. Baetidae: 128. Baetodes. 129. Callibaetis. 130. Cloeodes. 131. Mayobaetis. 132. Prebaeatodes. 133. Nanomis. (Escala= $1 \mathrm{~mm}$ ). 


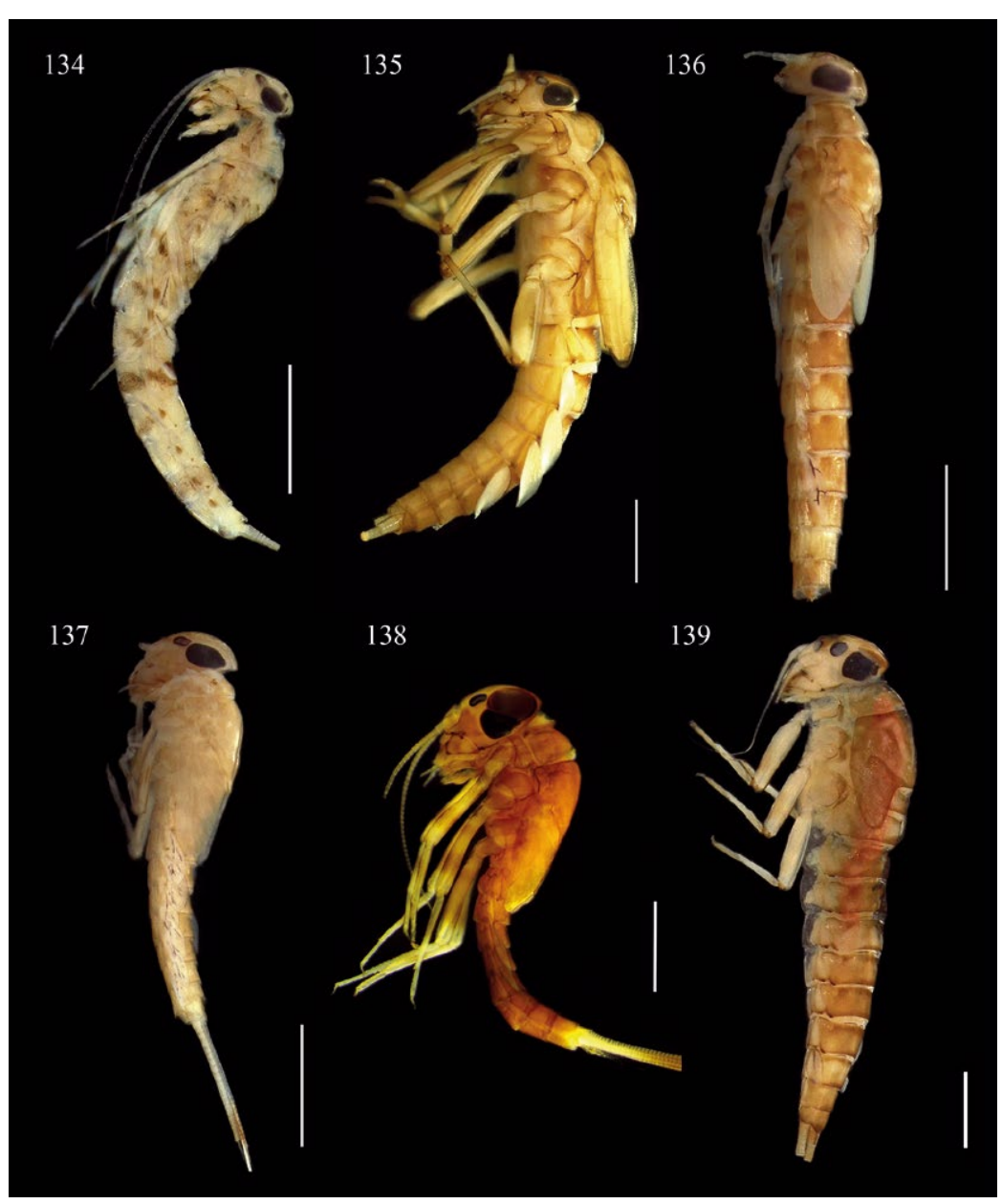

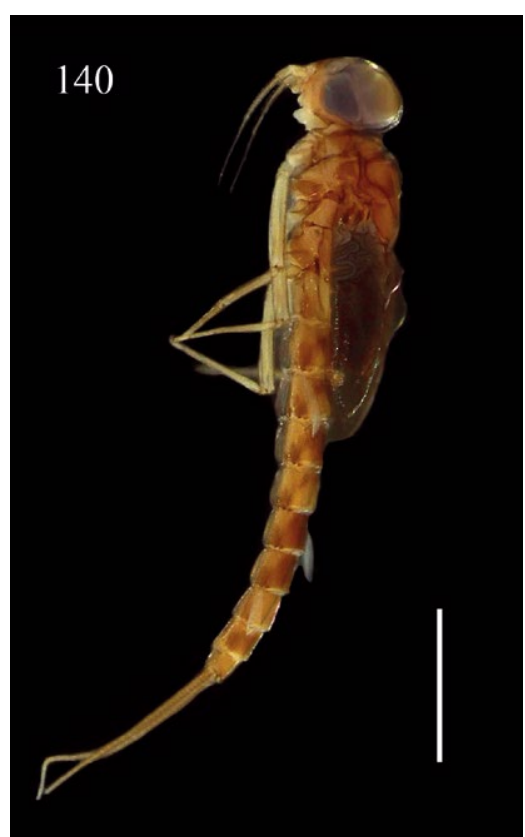

Figura 140. Baetidae: Zelusia. (Escala= $1 \mathrm{~mm}$ )

Figuras 134-139. Baetidae: 134. Waltzoyphius. 135. Andesiops. 136. Aturbina. 137. Cryptonympha. 138. Guajirolus. 139. Americabaetis. (Escala= $1 \mathrm{~mm})$.

\& Sartori 2009). Además, se destaca la alta riqueza de géneros respecto al estudio realizado en Madre de Dios por Sweeney et al. (2009), por el hallazgo de 13 géneros adicionales (Zelusia, Mayobaetis, Baetodes, Nanomis, Prebaetodes, Trichorytopsis, Lumahyphes, Yaurina, Haplohyphes, Euthyplocia, Simothraulopsis, Homoeoneuria y Hubbardipes).

\section{Literatura citada}

Allen R.K. 1973. New species of Leptohyphes Eaton (Ephemeroptera: Tricorythidae). The Pan-Pacific Entomologist. 49: 363-372.

Anderson E.P., A.C. Encalada, J.A. Maldonado-Ocampo, et al. 2011. Environmental flows: a concept for addressing effects of river alterations and climate change in the Andes. Pp. 326-338, in: Climate Change and Biodiversity in the Tropical Andes (SK Herzog, R Martínez, PM Jorgensen and H Tiessen, eds.). IAI-SCOPE, Sao Jose dos Campos.

Barber-James H.M., J.L. Gattolliat, M. Sartori, et al. 2008. Global diversity of mayflies (Ephemeroptera, Insecta) in freshwater. Pp. 339-350, in: Freshwater Animal Diversity Assessment (EV Balian, C Lévêque H Segers and K. Martens, eds.). Springer, Dordrecht. https://doi. org/10.1007/978-1-4020-8259-7_37
Barra L. 2015. Evaluación de la calidad del agua en nueve quebradas en el tramo carretero Puerto Maldonado-Mazuko, departamento de Madre De Dios, mediante el uso de macroinvertebrados acuáticos como indicadores biológicos. Tesis, Ingeniero forestal y Medio Ambiente. Facultad de Ingeniería. Universidad Nacional Amazónica de Madre de Dios. Acceso 07/05/2018

Boldrini R., F.F. Salles \& H.R. Cabette. 2009. Contribution to the taxonomy of the Terpides lineage (Ephemeroptera: Leptophlebiidae). Annales de Limnologie-International Journal of Limnology 45(4):219-229. https://doi. org/10.1051/limn/2009029

Brittain J.E. \& M. Sartori. 2009. Ephemeroptera:(Mayflies). Pp. 328-334, in: Encyclopedia of insects (VH Resh and RT Cardé, eds.). Academic Press, Oxford. https://doi. org/10.1016/B978-0-12-374144-8.00100-4

Buytaert W., R. Célleri, B. De Bièvre, et al. 2006. Human impact on the hydrology of the Andean páramos. Earth-Science Reviews 79(1-2):53-72. https://doi.org/10.1016/j. earscirev.2006.06.002

Coleman C.0. 2003. "Digital inking": How to make perfect line drawings on computers. Organisms Diversity and Evolution 3(4):303. https://doi.org/10.1078/14396092-00081 
Coleman C.O. 2006. Substituting time-consuming pencil drawings in arthropod taxonomy using stacks of digital photographs. Zootaxa 1360(1):61-68. https://doi. org/10.11646/zootaxa.1360.1.4

Cruz P.V. \& N. Hamada. 2019. A new species of Harpagobaetis Mol, with amended diagnosis of the genus, and new considerations on predatory species of Baetidae (Ephemeroptera) from South America. Zootaxa 4551(2):195210. https://doi.org/10.11646/zootaxa.4551.2.4

Cruz P.V., F.F. Salles \& N. Hamada. 2017. Additions and corrections to the systematics of mayfly species assigned to the genus Callibaetis Eaton, 1881 (Ephemeroptera: Baetidae) from South America. Zootaxa 4231(4):500534. https://doi.org/10.11646/zootaxa.4231.4.2

Cruz P. V., F.F. Salles \& N. Hamada. 2018. Contribution to the taxonomy of Paracloeodes Day 1955 (Ephemeroptera: Baetidae) from South America. Zootaxa 4403(1):1-49. https://doi.org/10.11646/zootaxa.4403.1.1

Dias L.G., C. Molineri \& P. Ferreira. 2007. Ephemerelloidea (Insecta: Ephemeroptera) do Brasil. Papeis Avulsos de Zoología 47(19):213-244. https://doi.org/10.1590/ S0031-10492007001900001

Domínguez E. 1988. Ecuaphlebia: A New Genus of Atalophlebiinae (Ephemeroptera: Leptophlebiidae) from Ecuador. Aquatic Insects 4:227-235. https://doi. org/10.1080/01650428809361334

Domínguez E. \& D.A. Dos Santos. 2014. Co-authorship networks (and other contextual factors) behind the growth of taxonomy of South American Ephemeroptera: a scientometric approach. Zootaxa 3754(1):59-85. https:// doi.org/10.11646/zootaxa.3754.1.3

Domínguez E. \& H.R. Fernández. 2009. Macroinvertebrados bentónicos sudamericanos. Sistemática y biología. Fundación Miguel Lillo, Tucumán, Argentina. 656pp. https://doi.org/10.1603/008.103.0401

Domínguez E., C. Molineri, C. Nieto, et al. 2019. Lista de especies de Ephemeroptera Sudamericanos. Recuperado de http://ibn-conicet.gob.ar/ephemeroptera-sudamericanos/

Domínguez E., C. Molineri, M. Pescador, et al. 2006. Ephemeroptera of South America. Aquatic Biodiversity in Latin America. Pensoft, Sofia. 646 pp.

Edmunds Jr G.F. 1948. A new genus of mayflies from western North America (Leptophlebiinae). Proceedings of the Biological Society of Washington 61:141-148. https:// biodiversitylibrary.org/page/34498581

Flowers R.W. \& C. De la Rosa. 2010. Ephemeroptera. Revista de Biología Tropical 58(Suppl 4) :63-93. https://doi. org/10.15517/rbt.v58i4

Grant P.M. 2001. Mayflies as food. Pp. 107-124, in: Trends in Research in Ephemeroptera and Plecoptera (E Domínguez, ed.). Springer, Boston. https://doi. org/10.1007/978-1-4615-1257-8_14

Gutiérrez Y. \& L. Días. 2015. Ephemeroptera (insecta) de Caldas, claves taxonómicas para los géneros y notas sobre su distribución. Papéis Avulsos de Zoologia 55(2):13-46. https://doi.org/10.1590/0031-1049.2015.55.02

Hubbard M.D. 1982. Catálogo abbreviado de Ephemeroptera da América do Sul. Papéis Avulsos de Zoologia 34:257282.

Jacobsen D. 2008. Tropical high-altitude streams. Pp. 219-256, in: Tropical stream ecology (D Dudgeon, ed.). Academic Press, Hong Kong SAR. https://doi.org/10.1016/ b978-012088449-0.50010-8
Jacobsen D. \& R. Marín. 2008. Bolivian Altiplano streams with low richness of macroinvertebrates and large diel fluctuations in temperature and dissolved oxygen. Aquatic Ecology 42(4): 643-656. https://doi.org/10.1007/ s10452-007-9127-x

Kluge N.J. 2007. A new taxon Hermanellonota, or subtribe Hermanellini subtr. n.(Ephemeroptera: Leptophlebiidae: Hagenulini), with description of three new species from Peruvian Amazonia. Russian Entomological Journal 16(4):385-400.

Lima L.R., C. Molineri, U. Pinheiro, et al. 2016. Two new species of Caenis Stephens, 1835 (Ephemeroptera: Caenidae) from South America. Zootaxa 4170(1):114-124. https://doi.org/10.11646/zootaxa.4170.1.5

Mariano R. 2011. A new species of Paramaka Savage \& Domínguez, 1992 (Ephemeroptera: Leptophlebiidae: Atalophlebiinae) from Brazil. Zootaxa 3038(1): 45-50. https://doi.org/10.11646/zootaxa.3038.1.3

Melo A.S., L.M. Bini, \& P. Carvalho. 2006. Brazilian articles in international journals on Limnology. Scientometrics 67(2):187-199. https://doi.org/10.1556/ scient.67.2006.2.3

Menetrey N., B. Oertli, M. Sartori, et al. 2007. Eutrophication: are mayflies (Ephemeroptera) good bioindicators for ponds?. Pp. 125-135, in: Pond Conservation in Europe (B Oertli, R Céréghino, J Biggs, S Declerck, A Hull and $M$ Miracle, eds.). Springer, Dordrecht. https://doi. org/10.1007/s10750-007-9223-x

Miñano J.P. 2017. Composición y estructura de la comunidad de Ephemeroptera (Insecta) en quebradas de baja y de gran altitud, Cusco-Perú. Tesis, Biólogo, mención Zoología. Universidad Nacional Mayor de San Marcos. Acceso 10/05/2018

Molina C.I., F.M. Gibon, J. Pinto, et al. 2008. Estructura de macroinvertebrados acuáticos en un río altoandino de la Cordillera Real, Bolivia: variación anual y longitudinal en relación a factores ambientales. Ecología aplicada 7(12):105-116. https://doi.org/10.21704/rea.v7i1-2.365

Molineri C. 2001. A New Genus of Leptohyphidae (Insecta: Ephemeroptera). Pp. 337-345, in: Trends in research in Ephemeroptera and Plecoptera (E Domínguez, ed.). Springer, Boston. https://doi.org/10.1007/978-14615-1257-8_38

Molineri C. 2003. Revision of the South American Species of Leptohyphes Eaton (Ephemeroptera: Leptohyphidae) with a key to the nymphs. Studies on Neotropical Fauna and Environment 38(1):47-70. https://doi. org/10.1076/snfe.38.1.47.14031

Molineri C. 2004. Phylogeny of the Allenhyphes-Traverhyphes group (Ephemeroptera: Leptohyphidae), with new subgenera, species and combinations. Tijdschrift voor Entomologie 147:197-220.

MolineriC. \&M.delC.Zúñiga. 2004. Lumahyphes, a newgenus of Leptohyphidae (Ephemeroptera). Aquatic Insects 26(1):1930. https://doi.org/10.1076/aqin.26.1.19.35379

Nieto C. \& F.F. Salles. 2006. Revision of the genus Paracloeodes (Ephemeroptera: Baetidae) in South America. Zootaxa 1303:1-33. doi.org/10.11646/zootaxa.1303.1.1

ONERN. 1976. (en línea). Mapa Ecológico del Perú - Guía explicativa. Acceso 25/01/2019

Padial A.A., L.M. Bini \& S.M. Thomaz. 2008. The study of aquatic macrophytes in Neotropics: a scientometrical view of the main trends and gaps. Brazilian Journal of Biology 68(4):1051-1059. https://doi.org/10.1590/s151969842008000500012 
Pescador M.L., M.D. Hubbard \& M.delC. Zúñiga. 2001. The status of the taxonomy of the mayfly (Ephemeroptera) fauna of South America. Pp. 37-42, in: Trends in research in Ephemeroptera and Plecoptera (E Domínguez, ed.). Springer, Boston. https://doi.org/10.1007/978-14615-1257-8_6

Pinto K. 2018. Influencia del bosque ribereño en la estructura y composición de la comunidad de macroinvertebrados bentónicos en nueve quebradas como bioindicadores - Madre de Dios. Tesis, Ingeniero forestal. Universidad Nacional de San Antonio Abad del Cusco. Acceso $13 / 07 / 2018$

Salles F.F. \& C. Molineri. 2006. Amanahyphes saguassu, a new genus and species of Leptohyphidae (Ephemeroptera: Ephemerelloidea) from northern Brazil. Aquatic Insects 28(1):1-12. https://doi. org/10.1080/13682820500343180

Salles F.F., E. Domínguez, C. Molineri, et al. 2018. Order Ephemeroptera. Pp. 61-117, in: Thorp and Covich's Freshwater Invertebrates (N Hamada, JH Thorp and DC Rogers, eds.). Academic Press, London. https://doi. org/10.1016/b978-0-12-804223-6.00003-2
Santos N.C.L., H.S.Santana, R.M. Dias, et al. 2016. Distribution of benthic macroinvertebrates in a tropical reservoir cascade. Hydrobiologia 765:265-275. https://doi. org/10.1007/s10750-015-2419-6

Sartori M. \& J.E. Brittain. 2015. Order Ephemeroptera. Pp. 873891, in: Thorp and Covich's Freshwater Invertebrates (J Thorp and DC Rogers, eds.). Academic Press, London. https://doi.org/10.1016/b978-0-12-3850263.00034-6

STROUD. 2010. (en línea) Leaf Pack Manual. Acceso 06/02/2018

Sweeney B.W., R.W. Flowers, D.H. Funk, et al. 2009. Mayfly communities in two Neotropical lowland forests. Aquatic Insects 31(supp1):311-318. https://doi. org/10.1080/01650420902833863

\begin{abstract}
Agradecimientos:
A Carlos Molineri, Wills Flowers y revisores anónimos por sus apreciables sugerencias al manuscrito. A Bernard Sweeney, David Funk y Wills Flowers quienes proporcionaron el valioso material biológico de la colecta manual. A Wills Flowers por la corroboración de algunos géneros de Leptophlebiidae. Al ingeniero Therany Gonzales y su equipo, quienes realizaron las colonizaciones con la metodología de paquete de hojas. A Lizeika Barra y Karen Pinto por cedernos el material biológico producto de su tesis. A Carmen Chávez, Directora de Programas en Perú (ACEER), cuya excelente gestión hizo posible el proyecto "Impacto de la Carretera Interoceánica sobre la calidad del agua en la región de las nacientes Andino-Amazónicas del este, Cusco-Madre de Dios, Perú" de donde se obtuvo la mayor parte de las muestras de éste estudio.
\end{abstract}

\section{Conflicto de intereses:}

Los autores no incurren en conflictos de intereses.

Rol de los autores:

PM realizó la identificación, redacción, elaboración de la clave taxonómica y esquemas. MO realizó la redacción, fotografía, mapa y edición. $\mathrm{AH}$ realizó la identificación, revisión y aprobación del manuscrito.

Fuentes de financiamiento:

Los autores declaran que no recibieron un financiamiento específico para esta investigación.

Aspectos éticos / legales:

Servicio Nacional Forestal y de Fauna Silvestre (SERFOR) del Ministerio de Agricultura y Riego - permiso de recolección No. 0235-2012-AGDGFFS-DGEFFS. 